\title{
An Asteroid Resource Map for Near-Earth Space
}

\author{
J.P. Sanchez ${ }^{1}$ \\ University of Strathclyde, Glasgow, Scotland G1 1XH, United Kingdom \\ C.McInnes ${ }^{2}$ \\ University of Strathclyde, Glasgow, Scotland G1 1XH, United Kingdom
}

\begin{abstract}
Most future concepts for the exploration and exploitation of space require a large initial mass in low Earth orbit. Delivering this required mass from the Earth's surface increases cost due to the large energy input necessary to move mass out of the Earth's gravity well. An alternative is to search for resources in-situ among the near Earth asteroid population. The near Earth asteroid resources that could be transferred to a bound Earth orbit are determined by integrating the probability of finding asteroids inside the Keplerian orbital element space of the set of transfers with an specific energy smaller than a given threshold. Transfers are defined by a series of impulsive maneuvers and computed using the patched-conic approximation. The results show that even moderately low energy transfers enable access to a large mass of resources.
\end{abstract}

\section{Nomenclature}

$a=$ semi-major axis of an orbit, $\mathrm{AU}$

$\mathrm{D}=$ asteroid diameter, $\mathrm{km}$ or $\mathrm{m}$

$e \quad=\quad$ eccentricity of an orbit

$g()=$. probability to find $k$ asteroids within a population of $n$

$\mathrm{H} \quad=\quad$ absolute magnitude

$i=$ inclination of an orbit, deg or rad

$k=$ number of asteroids successfully found in the accessible region

$m \quad=\quad$ mass of the asteroid, $\mathrm{kg}$

$M \quad=\quad$ mean anomaly of an orbit, deg or rad

MOID $=$ Minimum Orbital Intersection Distance, AU

$\mathrm{MOID}_{\text {cap }}=$ maximum MOID at which direct capture is possible with given $\Delta v_{\text {thr }}, \mathrm{AU}$

1 jpau.sanchez@strath.ac.uk, Research Fellow, Advanced Space Concepts Laboratory, Department of Mechanical Engineering, University of Strathclyde, Glasgow

2 colin.mcinnes@strath.ac.uk, member AIAA, Professor, Advanced Space Concepts Laboratory, Department of Mechanical Engineering, University of Strathclyde, Glasgow 


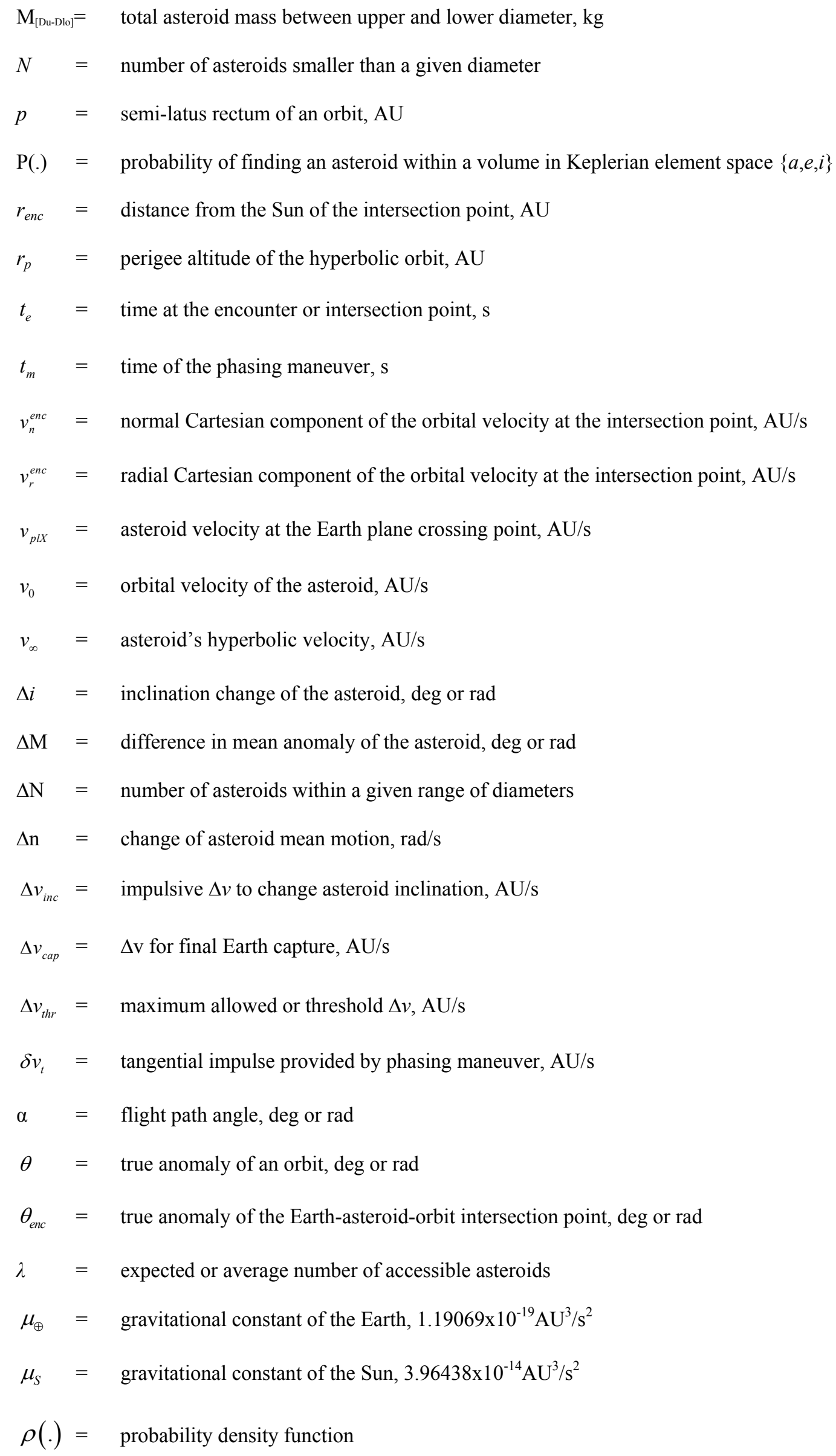




$$
\begin{aligned}
& \rho_{a}=\text { asteroid density, } \mathrm{kg} / \mathrm{m}^{3} \\
& \Omega \quad=\text { argument of the ascending node of an orbit, deg or rad } \\
& \omega \quad=\quad \text { argument of the periapsis of an orbit, deg or rad } \\
& \omega_{M O I D 0}=\text { argument of the periapsis yielding MOID equal to } 0, \mathrm{deg} \text { or rad } \\
& \omega_{\oplus}=\text { Earth's mean angular velocity, } \mathrm{rad} / \mathrm{s}
\end{aligned}
$$

\section{Subscripts}

$1 \mathrm{imp}=$ referring to the one impulse transfer

$2 \mathrm{imp}=$ referring to the two impulse transfer

\section{Introduction}

Most of the plausible futures for human space exploration and exploitation involve a large increase of mass in Earth orbit. Examples include space solar power, space tourism or more visionary human space settlements. Whether this mass is water for crew, propellant for propulsion or materials for structures, delivering the required mass from the Earth's surface may prove economically unfeasible due to the large energy input required to transport these resources to space. An alternative that has been widely discussed is to search among the population of asteroids in search of the required reservoir of material $[1,2]$.

Asteroids are of importance in uncovering the formation, evolution and composition of the solar system. In particular, near Earth asteroids (NEA) have rose in prominence because of two important points: they are among the easiest celestial bodies to reach from the Earth and they may represent a long-term threat [3]. The growing interest in these objects has translated into an increasing number of missions to NEA, such as the sample return missions Hayabusa [4] and Marco Polo [5], impactor missions such as Deep Impact $^{3}$ and possible deflector demonstrator missions such as Don Quixote ${ }^{4}$.

With regard to asteroid deflection, a range of methods have been identified to provide a change in the asteroid linear momentum [6]. Some of these methods, such as the kinetic impactor have been deemed to have a high technology readiness level (TRL), while others may require considerable development. Considering that the capability to impact an asteroid already exists (e.g., Deep Impact), or that the capability to deflect an asteroid may be available in the near future, a resource-rich asteroid could in principle be maneuvered and captured into a bound Earth orbit through judicious use of orbital dynamics. If direct transfer of the entire NEA is not

\footnotetext{
${ }^{3} \mathrm{http}: / /$ www.nasa.gov/mission_pages/deepimpact/main/index.html

${ }^{4} \mathrm{http}: / /$ www.esa.int/SPECIALS/NEO/SEMZRZNVGJE_0.html
} 
possible, or necessary, extracted resources could still be transferred to a bound Earth for utilization. It is envisaged that NEA could also be 'shepherded' into easily accessible orbits to provide future resources.

The main advantage of asteroid resources is that the gravity well from which materials would be extracted is much weaker than that of the Earth or the Moon. Thus, these resources could in principle be placed in a weaklybound Earth orbit for a lower energy cost than material delivered from the surface of the Earth or Moon. The question that arises is how much near-Earth asteroid material is there which could be captured with a modest investment of energy. This paper will attempt to answer this question by analyzing the volume of Keplerian orbital element space from which Earth can be reached under a certain energy threshold and then mapping this analysis to a reliable near Earth asteroid population model. The resulting resource map provides a realistic assessment of the material resources of near Earth space as a function of energy investment. It will be shown that there are substantial materials resources available at low energy based on the statistical distribution of near Earth asteroids.

The population of near-Earth objects is modelled in this paper by means of an object size distribution together with an orbital element distribution function. The size distribution is defined via a power law relationship between the asteroid diameter and the total number of asteroids with size lower than this diameter [7]. On the other hand, the orbital distribution used in this paper will rely on Bottke et al. [8] asteroid model to estimate the probability to find an object with a given set of Keplerian elements.

The dynamic model used to study the Keplerian orbital element space $\{a, e, i\}$ of asteroid-to-Earth transfers assumes a circular Earth orbit with a 1 AU semi-major axis. The Sun is the central body for the motion of the asteroid, and the Earth's gravity is only considered when the NEA motion is in close proximity. Since the orbital transfers will be modeled as a series of impulsive changes of velocity, for some conditions, analytical formulae relate the total change of velocity with the region of Keplerian space that can be reached.

Two different transfer models were considered for this study. Firstly, a phase-free two-impulse transfer, which is composed of a change of plane manoeuvre and a perigee capture manoeuvre at Earth encounter that would insert the asteroid, or extracted resources, in a weakly-bounded orbit around the Earth. This transfer, as with a Hohmann transfer analysis, provides a good conservative estimate of the exploitable asteroid material. Secondly, a phase-free one-impulse transfer, which only considers a perigee capture manoeuvre during the Earth fly-by. In this second case, only orbits that have initially very low Minimum Orbital Intersection Distances (MOID) can be captured. MOID is defined as the minimum possible distance between the Earth and the asteroid 
considering free-phasing for both objects. Finally, an estimation of the phasing manoeuvre required to meet the Earth at the orbital intersections will also be included on the transfer sequence.

\section{Near Earth Asteroid Distribution Model}

By convention, a celestial body is considered a Near Earth Object (NEO) if its perihelion is smaller than 1.3 AU and its aphelion is larger than 0.983 AU. This is a very broad definition which includes predominantly asteroids, but also a small percentage of comets (note that NEAs are a technically a subgroup of NEOs, although in this paper we may use the two acronyms as equivalent). NEOs are then the closest celestial objects to the Earth and therefore the obvious first targets for any resource exploitation mission (excluding the Moon).

The first NEA was discovered in 1898 (433 Eros) and since then more than 7000 asteroids have been added to the NEO catalogue. Most of these objects have been surveyed during the last 20 years as a consequence of the general recognition of the impact threat that these objects pose to Earth [9]. This recognition led to a series of efforts to catalogue $90 \%$ of objects with the potential to pose a global environmental threat [10] (i.e., Diameter $>1 \mathrm{~km})$. Subsequent recommendations suggested to pursue $90 \%$ completeness of the census of $140-\mathrm{m}$ objects by 2020 [7]. The new generation of surveys such as LSST [11] or Pan-STARRS [12] are well positioned to achieve this objective.

Together with the ever-growing catalogue of asteroids, the understanding of the origin and evolution of these objects has seen enormous advancements in recent years [13]. Still, it is not possible to know accurately the amount and characteristics of asteroid exploitable resources. However, reliable order of magnitude estimates may now provide some insight concerning the feasibility of future space resource exploitation and utilization concepts (e.g., space-based climate engineering $[14,15])$.

In order to determine the resource availability for future asteroid exploitation, a reliable statistical model of the near Earth asteroid population is required. The following section describes an asteroid model of the fidelity required for the subsequent analysis. The asteroid model described is composed of two parts; a population model, which describes the net number of asteroids as a function of object size and an orbit distribution model that describes the likelihood that an asteroid will be found in a given region of orbital element space.

\section{A. Near Earth Asteroid Population}

The main near Earth asteroid population model used here is taken from the Near-Earth Object Science Definition report [7]. It is based on the results of a substantial number of studies estimating the population of different ranges of object sizes by a number of techniques. The Near-Earth Object Science Definition report 
provides an accumulative population of asteroids that can be expressed as a constant power law distribution function of object diameter as:

$$
N(>D[k m])=C D^{-b}
$$

where $C=942$ and $b=2.354$ [7]. This constant power law distribution assumes that the average $1 \mathrm{~km}$ diameter object has an absolute magnitude $\mathrm{H}=17.75$. Figure 1, taken from Stokes et al. [7], shows in a logaritmic-scaled ordinate the population predictions of Eq.(1), as well as several previously published estimates and surveyed asteroids by january 2002 .

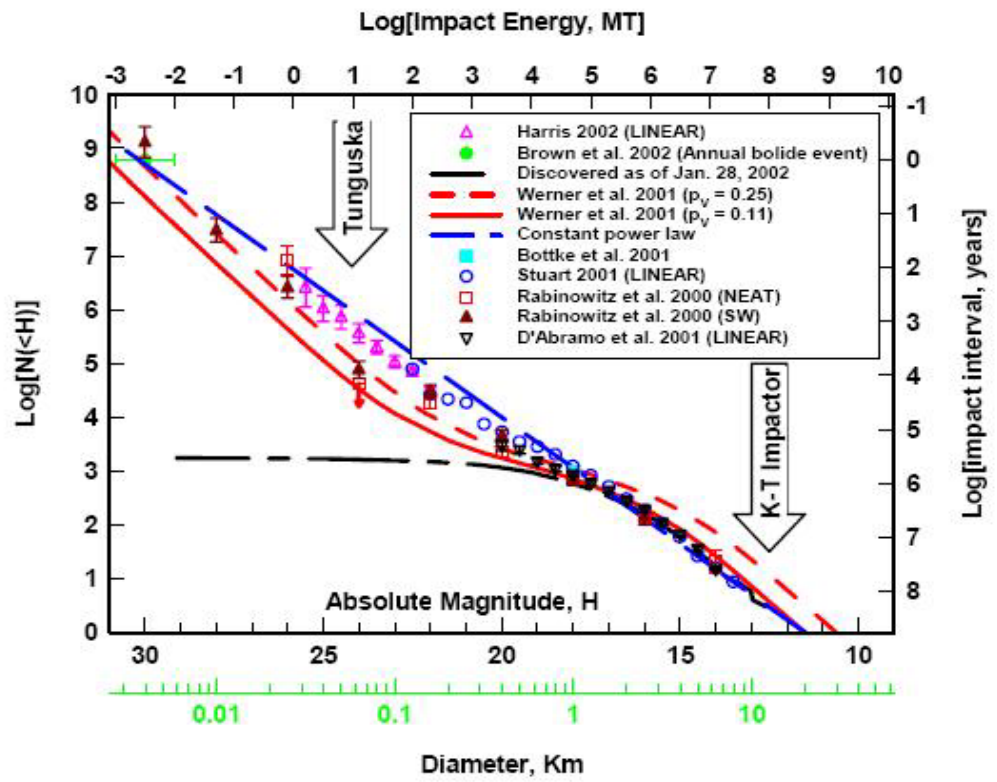

Figure 1: Hypothesized accumulative size distribution of Near Earth Objects, as well as different published estimates and surveyed asteroids by January 2002 (from Stokes et al [7]).

Assuming a population of asteroids defined by a power law distribution such as Eq. (1), one can easily calculate the total number of objects within an upper and lower diameter range:

$$
\Delta N\left(D_{\min }<D \leq D_{\max }\right)=C\left(D_{\min }^{-b}-D_{\max }^{-b}\right)
$$

where $D_{\max }$ and $D_{\min }$ are the maximum and minimum diameter chosen. An estimation of the total asteroid mass composed by all these objects can also be computed. To do so, the following integration needs to be performed:

$$
M_{\left[D_{\max }-D_{\min }\right]}=\int_{N_{D_{\max }}}^{N_{D_{\min }}} m \cdot d N(>D[\mathrm{~km}])
$$

where $m$ is the mass of the asteroid and $N_{D_{\min }}$ and $N_{D_{\max }}$ are the number of objects bigger than $\mathrm{D}_{\min }$ and $\mathrm{D}_{\max }$ respectively.

Assuming that all asteroids have a spherical shape and an average density $\rho_{a}$, the mass $m$ of the asteroid can be defined by $(\pi / 6) \cdot \rho_{a} \cdot D^{3}$ and the integration can be defined as an integration over the asteroid diameter: 


$$
M_{\left[D_{\max }-D_{\min }\right]}=\int_{D_{\max }}^{D_{\min }} \frac{\pi}{6} \cdot D^{3} \cdot \rho_{a} \cdot \frac{d N}{d D} d D
$$

where $d N / d D$ is the derivative of Eq. (1) with respect $D$. Integrating Eq. (4), the total mass of asteroid material composed of asteroids with diameters between $D_{\max }$ and $D_{\min }$ results in:

$$
M_{\left[D_{\max }-D_{\min }\right]}=\frac{\pi \cdot \rho_{a} \cdot C \cdot b}{6}\left(\frac{D_{\max }^{3-b}-D_{\min }^{3-b}}{3-b}\right)
$$

The average asteroid density $\rho_{a}$ can be approximated as $2600 \mathrm{~kg} / \mathrm{m}^{3}$ [16]. Thus, for example, Eq.(5) can yield the total mass of "Tunguska" size objects (i.e., from $50 \mathrm{~m}$ to $70 \mathrm{~m}$ diameter) in the near Earth space as being in the order of $1.6 \times 10^{14} \mathrm{~kg}$, while Eq.(2) estimates 600,000 of these objects [17]. More recent estimates of the population of small asteroids $[10,18]$ seem to indicate a possible drop by a factor of $2 / 3$ on the estimations given by Eq.(1) for small objects between 10 to 500 meters diameter. This new estimates can also be taken into account by using a four slope power law distribution adjusted accordingly. The total mass of "Tunguska" size objects using the four-slope power law decreases to $8.4 \times 10^{13} \mathrm{~kg}$.

Finally, if the maximum diameter is set equal to the largest near Earth object known, 1036 Ganymed, which is $32 \mathrm{~km}$ in diameter and the minimum object size is set at 1 meter diameter, Eq. (5) yields a total mass $4.4 \times 10^{16}$ kg. Note that this is only a very rough estimate provided by the assumption that the population of NEO follows a constant power law distribution such as Eq.(1) and that all objects are spherical with density $2600 \mathrm{~kg} / \mathrm{m}^{3}$. Still, this figure can be taken as a conservative and reliable order of magnitude estimate of the total asteroid mass in near Earth orbital space. It should be a conservative value since the three largest surveyed objects, Ganymed, Don Quixote and Eros, have already a combined total mass of order $5 \times 10^{16} \mathrm{~kg}$. It should also be reliable, since populations that can be modeled by power law distributions similar to Eq.(1) are ensured to have most of the accumulated mass built up by the largest members of the population. Eq.(2), for example, estimates that the three largest objects, which have diameters between 32 and $11 \mathrm{~km}$, sum up a mass of $2.2 \times 10^{16} \mathrm{~kg}$, already half of the total mass. Moreover, the four-slopes power law that account for the newest population estimates only yields a $5 \%$ decrease in the total mass, again due to the fact that the largest portion of the mass is composed by the largest objects, which are the same in the two estimates.

Hence, the population model described here provides a conservative order of magnitude for the total mass of objects, and allows reliable order of magnitude statistical estimate on the number and size of the undiscovered asteroids. 


\section{B. NEA Orbit Distribution}

This section describes the NEA (or NEO) orbital distribution model used to estimate the likelihood of finding an asteroid within a given volume of Keplerian orbital element space $\{\Delta a, \Delta e, \Delta i, \Delta \Omega, \Delta \omega, \Delta M\}$. This likelihood can also be interpreted as the fraction of asteroids within the specified region of the Keplerian space, and thus, if multiplied by Eq.(5), results in the portion of asteroid mass within that region. Hence, the ability to calculate this likelihood, together with the ability to define the regions of the Keplerian space from which the Earth can be reached with a given $\Delta v$ budget, will later allow us to compute the asteroid resources available in near-Earth space.

The NEA orbital distribution used here is based on an interpolation from the theoretical distribution model published in Bottke et al. [8]. The data used was very kindly provided by W.F. Bottke (personal communication, 2009). Bottke et al. [8] built an orbital distribution of NEA by propagating in time thousands of test bodies initially located at all the main source regions of asteroids (i.e., the $v 6$ resonance, intermediate source Marscrossers, the 3:1 resonance, the outer main belt, and the trans-Neptunian disk). By using the set of asteroids discovered by Spacewatch at that time, the relative importance of the different asteroid sources could be bestfitted. This procedure yielded a steady state population of near Earth objects from which an orbital distribution as a function of semi-major axis $a$, eccentricity $e$ and inclination $i$ can be interpolated numerically.

The remaining three Keplerian elements, the right ascension of the ascending node $\Omega$, the argument of periapsis $\omega$ and the mean anomaly $M$, are assumed here to be uniformly distributed random variables. The ascending node $\Omega$ and the argument of periapsis $\omega$ are generally believed to be uniformly distributed in near Earth orbit space [19] as a consequence of the fact that the period of the secular evolution of these two angles is expected to be much shorter than the life-span of a near Earth object [20]. Therefore, we can assume that any value of $\Omega$ and $\omega$ is equally possible for any NEA. All values of mean anomaly $M$ are also assumed to be equally possible, and thus $\mathrm{M}$ is also uniformly distributed between 0 to $2 \pi$.

A probability density function $\rho(a, e, i)$ has been created by linearly interpolating a 3-dimensional set of data containing the probability density at semi-major axes ranging from 0.05 to $7.35 \mathrm{AU}$ with a partition step size of $0.1 \mathrm{AU}$, eccentricity ranging from 0.025 to 0.975 with a partition step of 0.05 and inclination ranging from 2.5 to $87.5 \mathrm{deg}$ with a partition step of $5 \mathrm{deg}$. When $\rho(a, e, i)$ requires a value outside the given grid of points (e.g., inclination less than 2.5 degrees) then a nearest neighbor extrapolation is used for the dependence on semi-major axis and eccentricity, while a linear extrapolation is used for the dependence on inclination. Figure 2 shows both the $\rho(a, e, i)$ projected in the $\{a, e\}$ plane and the Aten, Apollo and Amor regions. 


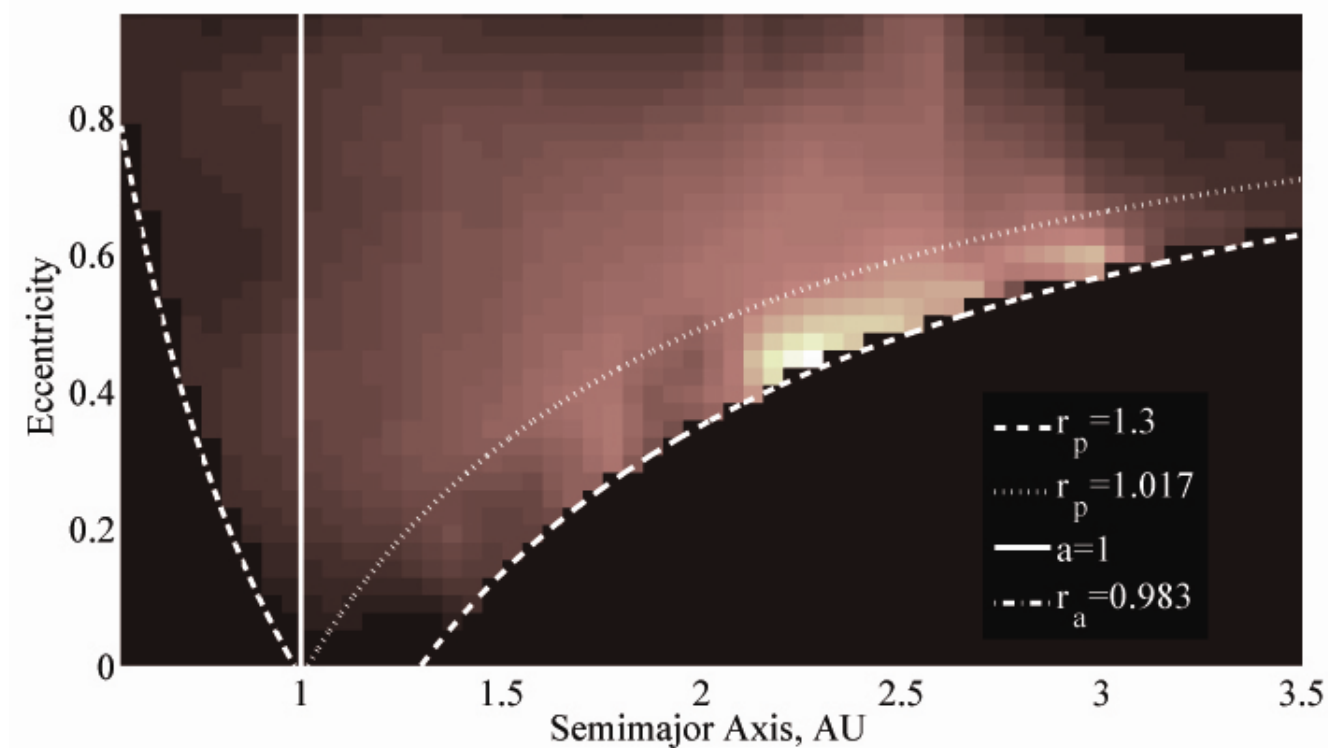

Figure 2: Bottke et al.[8] probability distribution built as an interpolation from the model data projected in the $\{a, e\}$ plane.

Finally, an integration such as:

$$
P=\int_{a_{\min }}^{a_{\max }} \int_{e_{\min }}^{e_{\max }} \int_{i_{\min }}^{i_{\max }} \rho(a, e, i) \cdot d i \cdot d e \cdot d a
$$

will then yield the probability of finding an asteroid within the Keplerian elements defined by $\left[a_{\max }, a_{\min }\right]$, $\left[e_{\max }, e_{\min }\right]$ and $\left[i_{\max }, i_{\min }\right]$. Section III will later describe how these limits can be defined as a function of the delta-velocity budget for different transfer types. The integration of a region of the Keplerian space, by means of Eq.(6), and multiplying this result by Eq.(2) provides a good estimation of the number of objects with diameters between $\left[D_{\max }, D_{\min }\right]$ that should be found within that region:

$$
\left\langle N_{\text {expected }}\right\rangle=P\left(\left[a_{\min }, a_{\max }\right],\left[e_{\min }, e_{\max }\right],\left[i_{\min }, i_{\max }\right]\right) \cdot N\left(D_{\min }>D<D_{\max }\right)
$$

When Bottke et al.[8] model was under development only 1000 near Earth objects were known, by $15^{\text {th }}$ April 2010, about 7000 objects have been surveyed. The NEA distribution model can then be tested by comparing the prediction of Eq.(7) against the real population. When doing so, care must be taken in comparing our expected population only with the fraction of the surveyed population that is complete or almost completed. The census of small asteroids $(<1 \mathrm{~km})$, which is not completed yet, suffers from observational effects (i.e., objects in orbits that can be more easily spotted are first discovered), and this implies that only the census of objects that are almost completed should be compared with the NEA distribution model. Eq.(1) foresees 861 objects bigger than $1 \mathrm{~km}$ diameter, currently there are 847 known objects with $\mathrm{H} \leq 17.75$, i.e., mean absolute magnitude of objects larger than $1 \mathrm{~km}$ diameter. Thus, we regard the survey of objects larger than $1 \mathrm{~km}$ diameter as almost completed, and therefore with little observation bias. Table 1 compares the observed population of 
asteroids with the expected population of NEA, calculated by means of Eq.(7). The table shows some difference in each one of the Keplerian regions checked, but in general the expected population matches pretty well the observed NEA and the differences can be regarded as statistical deviations from the mean.

Table 1. Comparison between NEA model predictions and surveyed population of asteroids larger than $1 \mathrm{~km}$

\begin{tabular}{|c|c|c|c|c|c|c|}
\hline & \multicolumn{2}{|c|}{ Expected } & \multicolumn{2}{|c|}{ Observed } & \multicolumn{2}{|c|}{ Difference } \\
\hline & $\mathrm{H} \leq 16$ & $\mathrm{H} \leq 17.75$ & $\mathrm{H} \leq 16$ & $\mathrm{H} \leq 17.75$ & $\mathrm{H} \leq 16$ & $\mathrm{H} \leq 17.75$ \\
\hline Total Asteroids & 129 & 861 & 184 & 847 & $-30 \%$ & $\sim$ \\
\hline \multicolumn{7}{|l|}{ Low Inclination } \\
\hline$<1$ deg & $\sim 1$ & $\sim 7$ & 1 & 3 & $0 \%$ & $133 \%$ \\
\hline$<5$ deg & $\sim 10$ & $\sim 68$ & 13 & 65 & $-23 \%$ & $5 \%$ \\
\hline$<10$ deg & $\sim 31$ & $\sim 205$ & 41 & 213 & $-24 \%$ & $2 \%$ \\
\hline$<20$ deg & $\sim 69$ & $\sim 455$ & 84 & 421 & $-18 \%$ & $8 \%$ \\
\hline \multicolumn{7}{|l|}{$v_{\infty}$} \\
\hline$<5 \mathrm{~km} / \mathrm{s}$ & $\sim 0$ & $\sim 0$ & 0 & 0 & $\sim$ & $\sim$ \\
\hline$<10 \mathrm{~km} / \mathrm{s}$ & $\sim 3$ & $\sim 20$ & 1 & 15 & $200 \%$ & $33 \%$ \\
\hline$<20 \mathrm{~km} / \mathrm{s}$ & $\sim 32$ & $\sim 211$ & 31 & 177 & $3 \%$ & $19 \%$ \\
\hline
\end{tabular}

\section{Asteroid to Earth Transfers}

This section will now describe the methodology followed to estimate the cost of transporting asteroid material to Earth. This paper envisages two broad scenarios for asteroid resource extraction and transportation. Firstly, raw or processed resources could be mined and transported from the asteroid to Earth for subsequent utilization. As an alternative, the entire asteroid could be transported to a bound Earth orbit where it would be processed. The first scenario, the transport of mined material, requires less energy to transport resources, since less mass is transported to Earth orbit, while requires that the mining operations occur in-situ. The latter requirement results in either very long duration manned missions, with the complexity that this entails, or, if the mining is performed robotically, the need for advanced autonomous systems due to both the communication delay between asteroid and Earth and the complexity of mining operations. The second scenario, on the other hand, requires moving a large mass, with the difficulty that this involves, but allows more flexible mining operations on the Earth's neighborhood. Both of these scenarios imply completely different mission architectures, although the optimality of each scenario is difficult to assess at this stage, and would ultimately depend on the technology readiness level of each individual technology (transfer and resource extraction) as well as the orbit of each particular asteroid or resource to exploit. In the remainder of this paper the term asteroid transfer will refer indistinctively to both of the aforementioned scenarios. In fact, the use of $\Delta v$ as a mapping parameter provides an estimation of the required scale of engineering only once the mass to be transported is also determined, and it is only here that the two possible scenarios differ. 
The transportation costs are then estimated through two different transfer types. The first transfer assumes a two-impulse trajectory, which includes a change of plane and Earth insertion maneuvre. While all Earthcrossing asteroids can be transferred to Earth using these two maneuvers, some asteroids have orientations such that they can easily fly-by the Earth with an almost negligible phasing maneuvre (i.e., asteroids with very low MOID). These objects could be captured with one single impulsive maneuvre during the Earth passage, i.e., one-impulse trajectory. These two transfer models will be defined by using a patched conic approximation, thus, the motion of the asteroid, or any material resources extracted, would be dominated by the gravitational influence of the Sun, except when in a very close encounter with the Earth. The Earth is also assumed to be in a circular orbit with radius $1 \mathrm{AU}$.

The transfer models are described as phase-free transfers. This means that the real orbital position is not taken into account, but only the geometry of the orbits is considered. Clearly, in order for an asteroid to meet the Earth during its orbital motion, not only the MOID must be very small (i.e., geometric consideration), but also Earth and asteroid must be found at the MOID point. Thus, an additional maneuvre will finally be considered in order to provide the gentle push necessary to render the required phasing.

\section{A. Two-impulse transfer}

The first impulsive maneuvre in this capture sequence provides the change of plane necessary to make the asteroid orbit coplanar with the Earth. Using a more complex and realistic sequence of maneuvers, a single combined maneuvre could provide both the required phasing and the change of plane such that an Earth flyby occurs. In that case, the asteroid transfer to Earth would not need to be strictly coplanar with the Earth and the change of velocity necessary for the maneuvre would be minimized. Unfortunately, this procedure would require a full numerical optimization for each individual case, which would be unmanageable for the scope of this paper.

A simpler approach is to consider a change of plane maneuvre such as:

$$
\Delta v_{i n c}=2 v_{p l X} \cdot \sin \left(\frac{\Delta i}{2}\right)
$$

where $\Delta v_{i n c}$ is the impulsive change of velocity necessary to change the orbital plane by $\Delta i$, and $v_{p l X}$ is the velocity of the asteroid at the Earth-orbital plane crossing. After the $\Delta v_{i n c}$ manoeuvre, the magnitude of the orbital velocity $v_{p l X}$ remains the same, only the inclination of the orbit has changed. Equation (8) allows a more analytical approach to the problem and at the same time provides a worst case scenario for the cost of the change of plane. 
The velocity of the asteroid at the Earth crossing plane $v_{p l X}$ will vary with the geometry and orientation of the asteroid orbit, i.e., $a$ and $e$, and argument of the periapsis $\omega$. As it has been previously discussed, the asteroid population model assumes that the argument of the periapsis $\omega$ behaves as a stochastic variable. As a consequence, and since the purpose of this model is to assess the asteroid resources that could be captured at the Earth, $v_{p l X}$ is defined as the velocity of the asteroid at its semi-latus rectum $p$, which again yields the worst case scenario for the change of plane maneuver for each orbital geometry. By doing so, Eq.(8) becomes a function only of the semi-major axis $a$, eccentricity $e$ and inclination $i$ (i.e., $\Delta i=i-i_{\oplus}=i$ )of the asteroid and can be written as:

$$
\Delta v_{i n c}(a, e, i)=2 \sqrt{\frac{\mu_{S}}{p}\left(1+e^{2}\right)} \cdot \sin \left(\frac{i}{2}\right)
$$

\section{Coplanar motion}

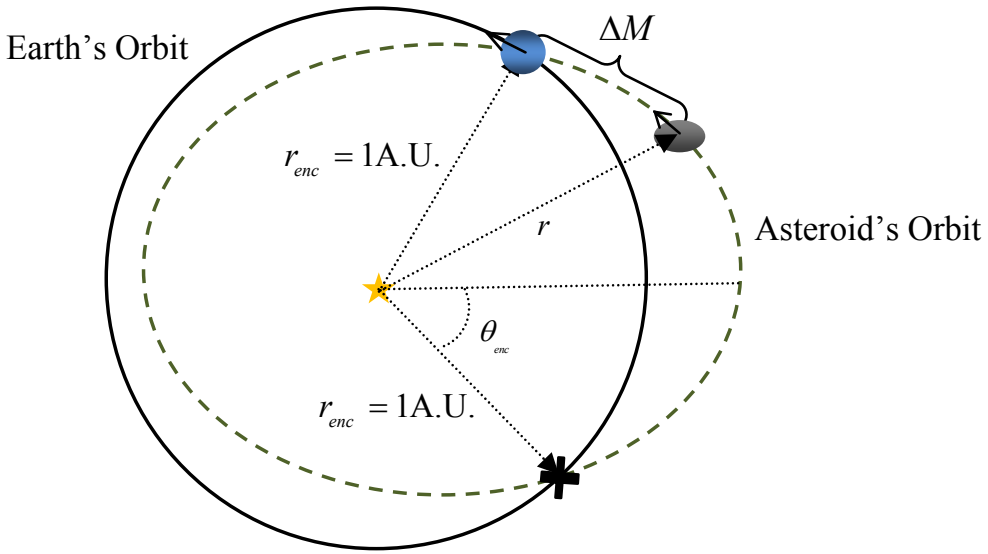

Figure 3: Orbital geometry of the coplanar model.

As shown in Figure 3, an asteroid in the ecliptic plane has two intersections (points of MOID equal 0) with the Earth's orbit. These are found when the asteroid is at 1AU from the Sun. Since the distance $r$ from the Sun to the asteroid is known, the equation of the orbit in polar coordinates yields the true anomaly of the two encounters $\theta_{e n c}$ :

$$
\theta_{e n c}= \pm \cos ^{-1}\left(\frac{p-1}{e}\right)
$$

where $p=a\left(1-e^{2}\right)$ is the asteroid's semi-latus rectum and the unit length in Eq. (10), and any of the following formulas in this paper, has been normalized to $1 \mathrm{AU}$. 
With the true anomaly of the encounter $\theta_{\text {enc }}$ known, the velocity at the encounter can now be defined by using the normal and radial components of Keplerian orbital motion:

$$
\begin{gathered}
v_{r}^{e n c}=\sqrt{\frac{\mu_{S}}{p}} e \sin \left(\theta_{\text {enc }}\right) \\
v_{n}^{e n c}=\sqrt{\frac{\mu_{S}}{p}}\left(1+e \cos \left(\theta_{\text {enc }}\right)\right)
\end{gathered}
$$

where $v_{r}{ }^{e n c}$ and $v_{n}{ }^{e n c}$ are the radial and normal velocity at the MOID point. Using Eq.(10), Eqs.(11) and (12) can be rewritten in a more suitable form as:

$$
\begin{gathered}
v_{r}^{e n c}=\sqrt{\frac{\mu_{S}}{p}\left(e^{2}-(p-1)^{2}\right)} \\
v_{n}^{e n c}=\sqrt{\mu_{S} p}
\end{gathered}
$$

Whenever the Earth-coplanar asteroid meets the Earth at $\theta_{\text {enc }}$, the velocity of the asteroid relative to the Earth will then be $\left(v_{r}^{e n c}, v_{n}^{e n c}-\omega_{\oplus} \cdot r_{\text {enc }}\right)$ with the Earth moving at an angular velocity $\omega_{\oplus}=\sqrt{\mu_{\text {Sun }}}$. This Earth encounter conditions will result on a hyperbolic motion of the asteroid relative to the Earth with an excess velocity as:

Final Earth insertion

$$
v_{\infty}=\sqrt{\mu_{S}(3-1 / a-2 \sqrt{p})}
$$

If MOID is zero or almost zero, the Earth encounter could be easily tuned by a phasing manoeuvre so that the altitude during the Earth fly-by is some given minimum distance (chosen here to be 200km) above the Earth's surface. At this minimum altitude a final insertion manoeuvre could be performed.

The notion of targeting asteroids towards the Earth may raise some concerns with regards to a possible enhancement of the impact threat. Clearly, changing the orbit of a large NEA could potentially be a threat to Earth, although engineering the orbit of large objects may also be unfeasible. Thus for this objects transferring mined resources may provide the best and only option. On the other hand, for smaller bodies the impact hazard can be mitigated since bodies of tens of meters of diameter should completely ablate in the atmosphere [21]. Thus, bodies in the order of 10 meters diameter may be perfect targets for first capture demonstrator missions.

A parabolic orbit is assumed here to be the threshold between an Earth-bound orbit and an Earth escape orbit. Hence, the $\Delta v$ necessary for an Earth capture $\Delta v_{\text {cap }}$ at the perigee passage results in: 


$$
\Delta v_{\text {cap }}=\sqrt{\frac{2 \mu_{\oplus}}{r_{p}}+v_{\infty}^{2}}-\sqrt{\frac{2 \mu_{\oplus}}{r_{p}}}
$$

where $v_{\infty}$ is the hyperbolic excess velocity described in Eq.(15) and $r_{\mathrm{p}}$ is the pericentre altitude (i.e., $r_{\oplus}+200 \mathrm{~km}$

). Finally, the sum of Eqs.(9) and (16) provides the total $\Delta v$ budget for a two-impulse transfer to Earth.

\section{Keplerian Feasible Regions}

As noted earlier, the integration in Eq.(6) yields the probability of an asteroid to be found within a specified Keplerian region. By rearranging Eqs.(9) and (16), we can now define the regions from which transfers to Earth cost less than a given limit $\Delta v_{\text {thr }}$. Figure 4, for example, shows the Keplerian region in the plane $\{a, e\}$ where asteroid resources can be transfer to Earth with a total $\Delta v$ equal or lower than $2.37 \mathrm{~km} / \mathrm{s}$. This $\Delta v$ corresponds to the Moon's escape velocity, thus offering a direct comparison between material available at the Moon and within an equivalent energy threshold elsewhere in the solar system. Also, superimposed in the figure are almost 5,000 asteroids (tiny dots and small crosses), which had been surveyed by April 2010.

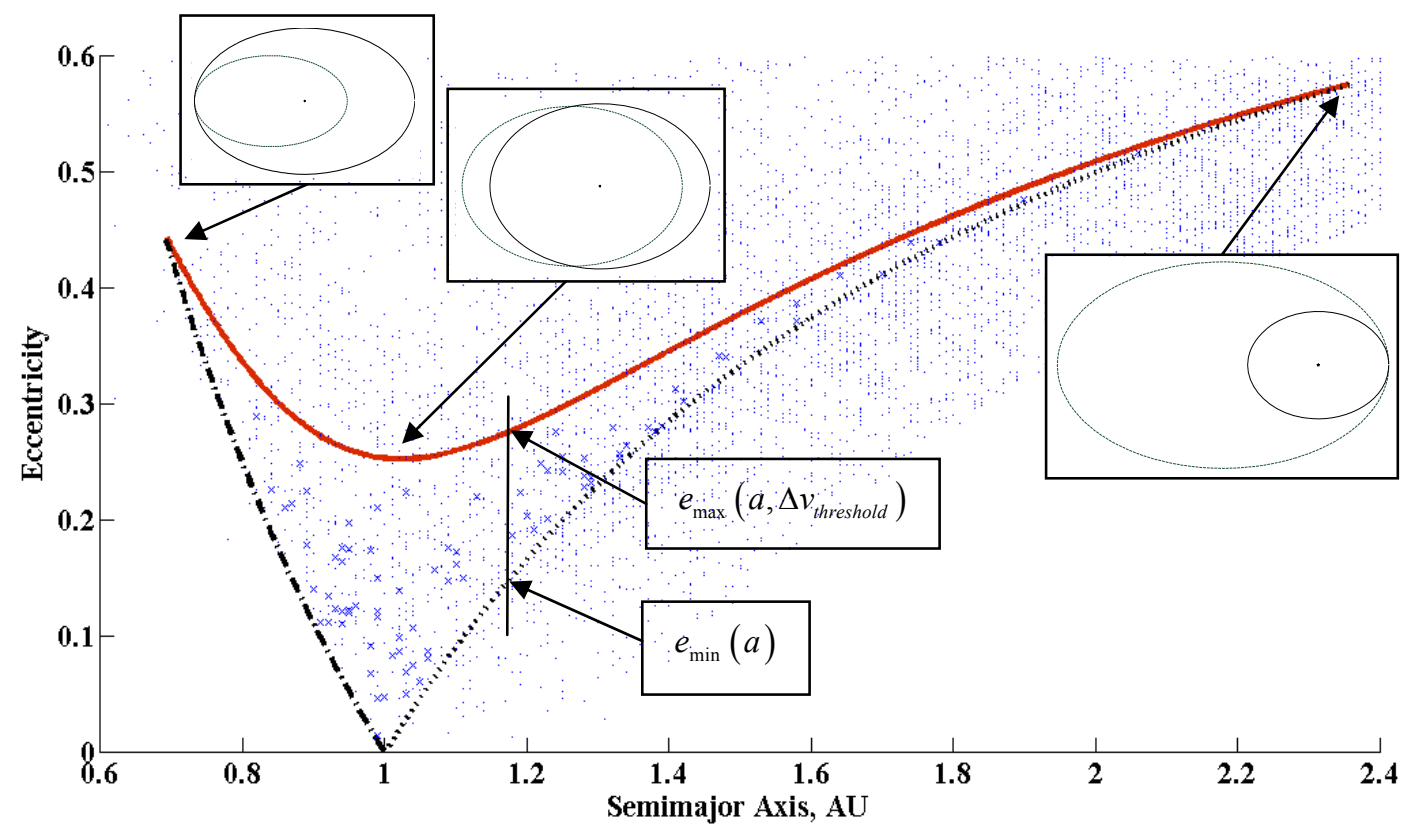

Figure 4. Keplerian \{a,e\} space reached by a maneuver of 2.37 km/s (i.e., Moon's escape velocity). Superimposed are all near Earth asteroids known within the \{a,e\} space as of April 2010.

Figure 4 shows three different lines (solid, dash-dotted and dotted line) delimiting an area in the $\{a, e\}$ plane. The solid line results from expressing Eq.(16) as an explicit function of the semi-major axis a and $\Delta v_{\text {cap }}$ necessary for an Earth capture:

$$
e\left(\Delta v_{c a p}, a\right)=\sqrt{1-\frac{1}{4 a}\left(3-\frac{1}{a}-\frac{v_{\infty}^{2}}{\mu_{S}}\right)^{2}}
$$

where the hyperbolic excess velocity $v_{\infty}$ is defined as: 


$$
v_{\infty}^{2}=\left(\Delta v_{c a p}+\sqrt{\frac{2 \mu_{\oplus}}{r_{p}}}\right)^{2}-\frac{2 \mu_{\oplus}}{r_{p}}
$$

Equation (17) therefore yields the value of eccentricity for which an asteroid with semi-major axis $a$ can be captured with a manoeuvre $\Delta v_{c a p}$ at the perigee passage. Asteroids with semi-major axis $a$, but eccentricity lower than the result provided by Eq. (17) should be captured with a manoeuvre lower than $\Delta v_{c a p}$. Thus, if $\Delta v_{c a p}$ is set to the maximum allowed manoeuvre $\Delta v_{\mathrm{thr}}$, the eccentricity resulting from Eq. (17) is also the maximum allowed eccentricity, $e_{\max }=e\left(\Delta v_{t h r}, a\right)$, i.e., solid line in Figure 4 when $\Delta v_{\text {thr }}=2.37 \mathrm{~km} / \mathrm{s}$.

Eccentricities lower than $e_{\max }$ require lower $\Delta v$ manoeuvres to be captured at the Earth, but there is a geometrical limit to the minimum Earth insertion manoeuvre $\Delta v_{c a p}$.The minimum $\Delta v_{c a p}$ occurs when the encounter geometry is such that the intersection is at the line of apsis. With this geometry only one intersection point exists, and lower eccentricities imply orbits with no Earth-crossing points (see Figure 4). The minimum allowed eccentricity for an orbit with semi-major axis $a$ is therefore:

$$
e_{\min }(a)=\left\{\begin{array}{l}
(1-1 / a) \text { if } a \geq 1 \\
(1 / a-1) \text { if } a<1
\end{array}\right.
$$

so that, if $a \geq 1$, the periapis radius is 1 (see dotted line in Figure 4), and, if instead $a<1$, the apoapsis is 1 (see dash-dotted line in Figure 4).

Once the analytical expressions for the maximum and minimum eccentricity $e_{\max }$ and $e_{\min }$ are known, the maximum and minimum allowed semi-major axis $a$ can be computed by finding when $e_{\max }\left(\Delta v_{t h r}, a\right)=e_{\min }(a)$ occurs. The latter equation results in a second degree polynomial with the following two solutions:

where $v_{\infty}$ is defined as in Eq.(18).

$$
a_{\min }\left(\Delta v_{t h r}\right)=\frac{1}{1-\frac{v_{\infty}^{2}}{\mu_{s}}+2 \sqrt{\frac{v_{\infty}^{2}}{\mu_{s}}}} \text { or } a_{\text {max }}\left(\Delta v_{t h r}\right)=\frac{1}{1-\frac{v_{\infty}^{2}}{\mu_{s}}-2 \sqrt{\frac{v_{\infty}^{2}}{\mu_{s}}}}
$$

Inside this delimited area within the $\{a, e\}$ Keplerian space, we can ensure that the coplanar capture maneuvers will be lower than the limit threshold. Thus, the reminder impulse, $\Delta v=\Delta v_{\mathrm{thr}}-\Delta v_{\mathrm{cap}}(a, e)$, can be used for changing the plane of any available object. Rearranging Eq.(9), the maximum object's inclination that can be modified and set into a coplanar motion with the Earth results: 


$$
i_{\max }\left(a, e, \Delta v_{t h r}\right)=2 \cdot \sin ^{-1}\left(\frac{\Delta v_{t h r}-\Delta v_{c a p}(a, e)}{2\left(\frac{\mu_{S}}{p}\left(1+e^{2}\right)\right)^{1 / 2}}\right)
$$

Finally, any object within the Keplerian volume defined by equations (17) to (21) will require a total $\Delta v$ lower than the limit threshold and some $\Delta v$ budget will still be available for phasing. Note that two types of symbols were used in Figure 4 to represent the surveyed asteroids. The small crosses represent all those objects that fall inside the volume calculated by the limits previously described when the Moon's escape velocity is used as $\Delta v_{t h r}$. Thus, dots falling inside the area delimited in Figure 4 have inclinations larger than the result of equation (21). As shown in the figure, a considerable number of surveyed objects have orbits from which material could be transferred to the Earth with a lower energy requirement than from the surface of the Moon. It is also important to remark that by April 2010 only the survey of objects larger than $1 \mathrm{~km}$ diameter is virtually completed, while less than $0.1 \%$ of the objects between 30 and 300 meters are known. Hence, an important portion of objects are still to be discovered.

\section{B. One-impulse transfer}

As shown in Figure 3, if the asteroid is coplanar with the Earth orbit, two orbital crossing points will always exist, as long as the periapsis and apoapsis of the asteroid's orbit are smaller and larger than 1 AU, respectively. On the other hand, if the asteroid is not coplanar with the Earth's orbit, only specific values of the angle of the periapsis $\omega$ will render an orbital intersection or a MOID small enough for a capture to be possible (see Figure 5).

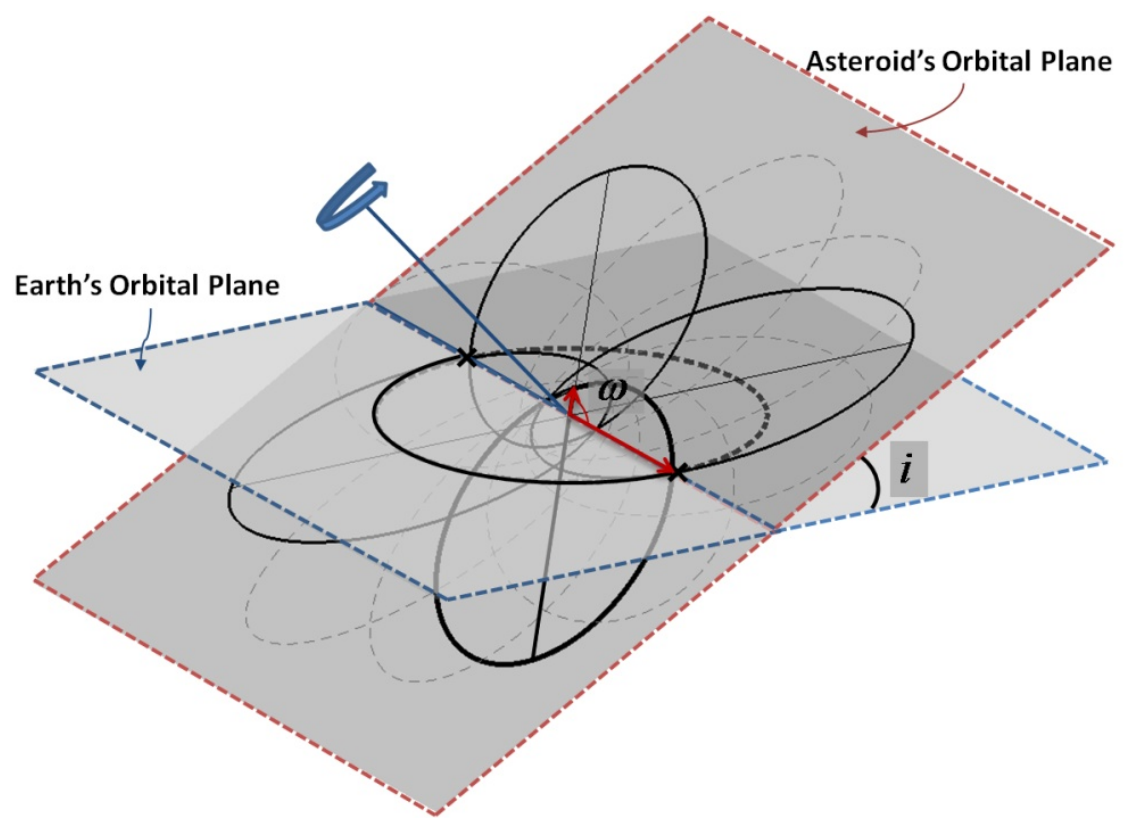

Figure 5: Representation of all possible orientations of an orbit as a function of argument of the periapsis w. The figure shows two orbital planes, one for the Earth orbit and one for the asteroid orbit. By continuously 
changing the argument of the periapsis, all possible orientations of the asteroid orbit in the plane are yielded. The two crosses mark the Earth orbital crossing points which are possible only for four different values of the argument of the periapsis $\omega$. Two arrows show the argument of the periapsis $\omega$ for one of the four configurations.

As shown by Figure 5, only 4 specific values of $\omega$ yield a MOID equal to zero (i.e., an intersection between the two orbits). Except if the semilatus rectum $p$ is equal to 1, in which case there will be only two values of $\omega$ yielding two simultaneous crossing points. Equation (10) already provided the two possible true anomalies that give the asteroid a distance of $1 \mathrm{AU}$ from the Sun. Therefore, for the orbital intersection to occur in the nonecliptic asteroid case, one of these two angles is required to coincide with the line of nodes, i.e., the straight line where the two orbital planes meet. This yields four different arguments of the periapsis $\omega$ for which the MOID is 0 :

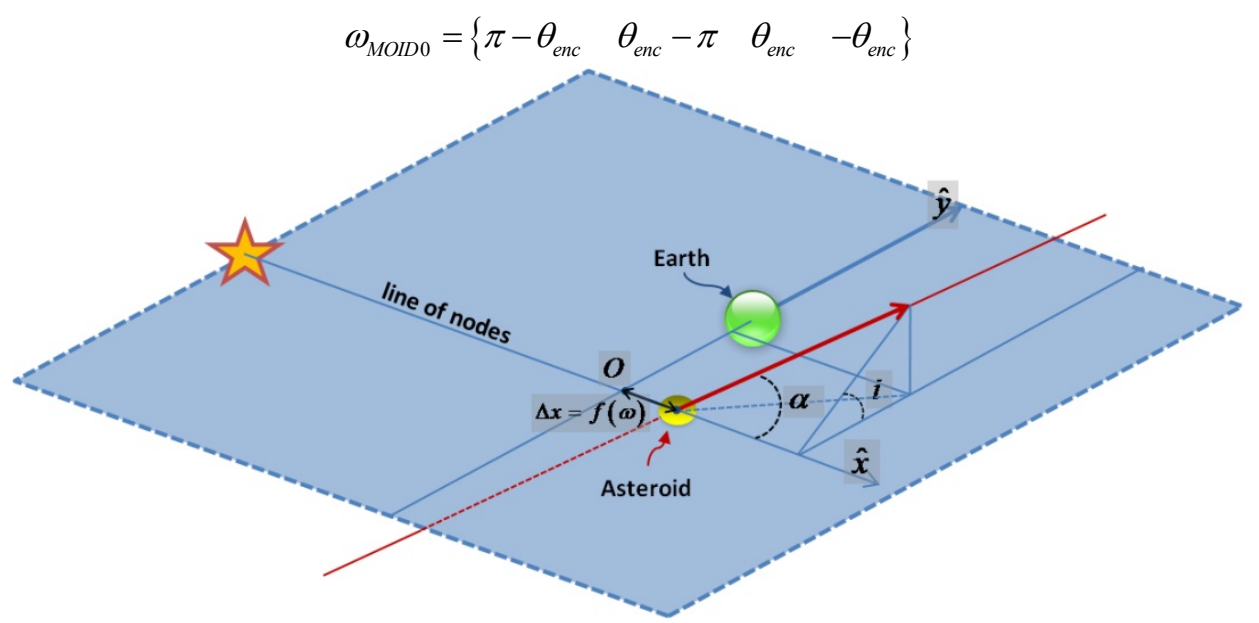

Figure 6: Set of coordinates used to compute Eq.(23).

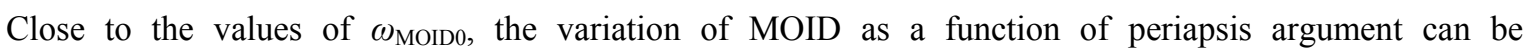
approximated linearly $[20,22]$. With the axis shown in Figure 6, the motion of the Earth and the asteroid can be well described using a linear approximation of the Keplerian velocities of the two objects for true anomaly $\theta_{\text {enc }}$. This defines two straight line trajectories, and thus, the minimum distance between these two linear trajectories can be found. The minimum distance can then be written as an explicit function of $\Delta x$ (i.e., distance between the centre of the coordinates described in Figure 6 and the point at which the asteroid crosses the Earth orbital plane), which can also be described as a linear function of the argument of the periapsis $\omega$. Finally, an expression such as:

$$
M O I D=\frac{\min \left[\left|\omega_{M O I D 0}-\omega\right|\right]}{\sqrt{\left(\frac{1}{\sin (i)}\right)^{2}+\tan ^{2}(\alpha)}}
$$


yields an approximate value of the MOID distance. The expression min[|.|] denotes the minimum value of the absolute differences with any of the angles $\omega_{\text {MOID } 0}$ and the tangent of the flight path angle can be calculated as:

$$
\tan (\alpha)=\frac{p}{\sqrt{e^{2}-(p-1)^{2}}}
$$

For a complete derivation of a similar formulae, the reader can refer to Opik's work[20] or alternatively to Bonanno's work [22]. Note that Eq.(23) is valid only for values of $\omega$ close to any of the values of $\omega_{\text {MOID } 0}$ from equation (22). Figure 7 show, as example, the evolution of the MOID distance as a function of periapsis argument for the elliptic orbit plotted in Figure 5(i.e., $\mathrm{a}=1.1 \mathrm{AU}, \mathrm{e}=0.8, \mathrm{i}=30^{\circ}$ ). The figure compares the results of MOID calculated by means of Eq.(23) with the results of a numerical algorithm that finds MOID by minimizing the distance between two positions defined by the true anomaly of each orbit. As it can be seen, Eq.(23) yields a very good approximation of the real MOID when MOID is small. Clearly, the error committed by this formulation increases for very low inclinations and very low eccentricities, but it is still tolerable for inclinations of 0.1 degree and eccentricities of 0.01 .

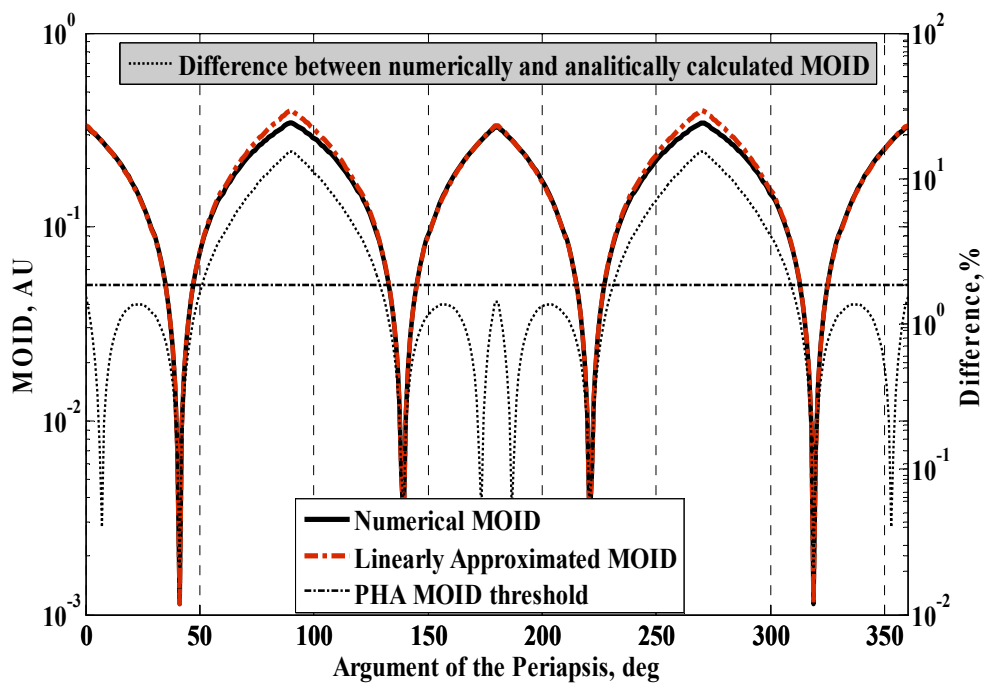

Figure 7: Comparison between the analytical and numerical approaches to compute MOID.

\section{Capture at MOID point}

Once it has been shown that the analytical approximation of MOID is a reliable way of assessing the distance between two orbits, we can define the maximum MOID at which the capture of an object is possible given a limiting $\Delta v$ budget. Eq.(16) defined the required Earth capture maneuver $\Delta v_{\text {cap }}$ as a function of the hyperbolic excess velocity $v_{\infty}$ and the pericenter altitude $r_{p}$. The latter can also be expressed as an explicit function of the hyperbolic velocity $v_{\infty}$ and the impulsive maneuver:

$$
r_{p}\left(\Delta v_{c a p}, v_{\infty}\right)=\frac{8 \mu_{\oplus} \Delta v_{c a p}^{2}}{\left(v_{\infty}^{2}-\Delta v_{c a p}^{2}\right)^{2}}
$$


Since equation (25) refers to non-coplanar asteroids, the hyperbolic velocity $v_{\infty}$ needs to be calculated as:

$$
v_{s}^{2}=\mu_{s}(3-1 / a-2 \sqrt{p} \cdot \cos (i))
$$

This expression can be derived by noticing that the relative velocity at the encounter for a non-coplanar asteroid can be expressed as $\left(v_{r}^{\text {enc }}, v_{n}^{\text {enc }} \cdot \cos (i)-\omega_{\oplus} \cdot r_{\text {enc }}, v_{n}^{e n c} \cdot \sin (i)\right)$.

Finally, in order to know the maximum MOID at which a direct capture is possible, the distance $r_{p}$ needs to be corrected by the hyperbolic factor, i.e., factor that accounts for the gravitational pull of the Earth during the asteroid's final approach to the Earth. This results on:

$$
\operatorname{MOID}_{c a p}=r_{p}\left(\Delta v_{c a p}, v_{\infty}\right) \sqrt{1+\frac{2 \mu_{\oplus}}{r_{p}\left(\Delta v_{c a p}, v_{\infty}\right) \cdot v_{\infty}^{2}}}
$$

Note that if the perigee altitude resultant from Eq.(25) is smaller than the radius of the Earth would mean that the capture of that particular body is not feasible under that particular $\Delta v$ threshold used. In fact, the feasible limit for a fly-by was set to $200 \mathrm{~km}$ altitude from the surface of the Earth, also to account for the Earth atmosphere.

Fraction of capturable asteroids

The previous section provided the means of calculating the MOID at which capture is possible as a function of $\Delta v_{\text {cap }}$. Using the linearly approximated MOID in Eq.(23), we can see that within a distance $\Delta \omega$ of $\omega_{\text {MOID } 0}$ such as:

$$
\Delta \omega=\operatorname{MOID}_{\text {cap }} \cdot \sqrt{\left(\frac{1}{\sin (i)}\right)^{2}+\tan ^{2}(\alpha)}
$$

a direct capture of the asteroid is possible, since the minimum orbital distance is ensured to be smaller than MOID cap, and thus, the capture impulse should be smaller than $\Delta v_{\text {cap }}$. One may think then that the total range at the neighborhood of $\omega_{\text {MOIDo }}$ is $2 \Delta \omega$, and since there are 4 different $\omega_{\text {MOIDo }}$, the total range of $\omega$ at which capture is possible should be $8 \Delta \omega$. This is generally correct, but attention must be pay when overlapping of ranges occurs. If the semilatus rectum $p$ is close two 1 , the values $\theta_{\text {enc }}$ and $\pi$ - $\theta_{\text {enc }}$ are also close and their ranges $\left(\theta_{e n c} \pm \Delta \omega\right.$ and $\left.\pi-\theta_{e n c} \pm \Delta \omega\right)$ may overlap, a correction is applied in those cases.

The fraction of asteroids with given $\{a, e, i\}$ that can be captured with a given $\Delta v$ budget is then:

$$
f(a, e, i)=\frac{8 \Delta \omega(a, e, i)}{2 \cdot \pi}
$$


without overlap correction. Fraction $f$ provides the portion of material with Keplerian elements $\{a, e, i\}$ that could be captured with a single maneuver $\left(\leq \Delta v_{\text {cap }}\right)$ at the Earth. Capture of asteroid material by means of only one impulse would simplify considerably the engineering challenges of implementing the two-impulse transfer, described in section III.A, since this type of transfer requires of a spacecraft to be sent to deep-space to perform a change of plane.

Keplerian Feasible Regions

The capture feasible regions using one-impulse transfers in the $\{a, e\}$ subspace are the same than in section III.A.3. The only difference between the feasible volume $\{a, e, i\}$ of the two-impulse and the one-impulse model lies in the inclination. Since no change of inclination is required, the maximum inclination from which asteroids can be captured is greatly increased. The limit threshold can be computed by realizing that $v_{\infty}^{2}$ calculated as in Eq.(26) must be equal to $v_{\infty}^{2}$ calculated as in Eq.(18), thus:

$$
i_{\max }(a, e, \Delta v)=\cos ^{-1}\left(\frac{1}{2 \sqrt{p}}\left(3-1 / a-\frac{v_{\infty}^{2}}{\mu_{S}}\right)\right)
$$

where $v_{\infty}$ is calculated as in Eq.(18) with an $r_{p}=r_{\oplus}+200 \mathrm{~km}$.

\section{Average Accessible Mass}

At this point, the transfer or material transport models can be combined with the NEO model to provide an estimate of the available material that could be exploited for future space ventures. The accessible asteroid material can now be mapped as a function of the limiting $\Delta v$ budget by using Eqs.(17) to (21) for two-impulse transfers and Eqs.(17) to (20) and (30) for one-impulse transfer. These equations delimit the set of $\{a, e, i\}$ from which an asteroid could be transported to a weakly-bound Earth orbit with a transfer requiring a total $\Delta v$ lower than a fixed threshold. The fraction of the near-Earth object population that falls into this feasible region can be calculated by integrating the NEO orbital distribution over the entire volume of feasible transfers. For a twoimpulse transfer model the fraction of NEO material as a function of $\Delta v_{t h r}$ is:

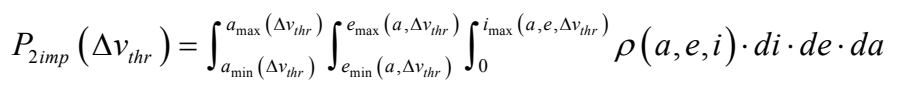

where the integrations limits are described by Eqs.(17) to (21). Note that, unlike the two-impulse transfer, for which any asteroid with a feasible set $\{a, e, i\}$ can be transferred to Earth, not all the asteroids with feasible $\{a, e, i\}$ can be transported by using a one-impulse transfer, but only the fraction $f(a, e, i)$ (Eq.(29)) of asteroids with orientation such that their MOID are smaller than MOID ${ }_{\text {cap. }}$. Hence, the fraction of the near-Earth object population that is accessible by means of one-impulse transfer and as a function of $\Delta v_{t h r}$ is: 


$$
P_{1 i m p}\left(\Delta v_{t h r}\right)=\int_{a_{\min }\left(\Delta v_{t h r}\right)}^{a_{\max }\left(\Delta v_{t h r}\right)} \int_{e_{\min }\left(a, \Delta v_{t h r}\right)}^{e_{\max }\left(a, \Delta v_{t h r}\right)} \int_{0}^{i_{\max }\left(a, e, \Delta v_{t h r}\right)} \rho(a, e, i) \cdot f(a, e, i) \cdot d i \cdot d e \cdot d a
$$

where the integrations limits are described by Eqs.(17) to (20) and (30).

When the fractions of NEO material $P$ are known, the average accessible mass can be calculated by multiplying the fraction of material by the total mass of the NEO population, Eq. (5). Figure 8 shows the accessible resources available considering all near Earth objects between $32 \mathrm{~km}$ (i.e., largest Near Earth object known today) and 1 meter diameter. This result may be refined by choosing the minimum asteroid diameter for useful resources exploitation as $10 \mathrm{~m}$ or $100 \mathrm{~m}$ diameter, instead of $1 \mathrm{~m}$, although these two lower boundaries barely modify the results shown in Figure 8 , since only $0.42 \%$ or $2.28 \%$ of the asteroid mass is made up by objects sized between 1-10 m or 1-100 m, respectively. Thus, the discussion of whether or not it is worth exploiting such small objects as $1 \mathrm{~m}$ diameter can be postponed at this point.

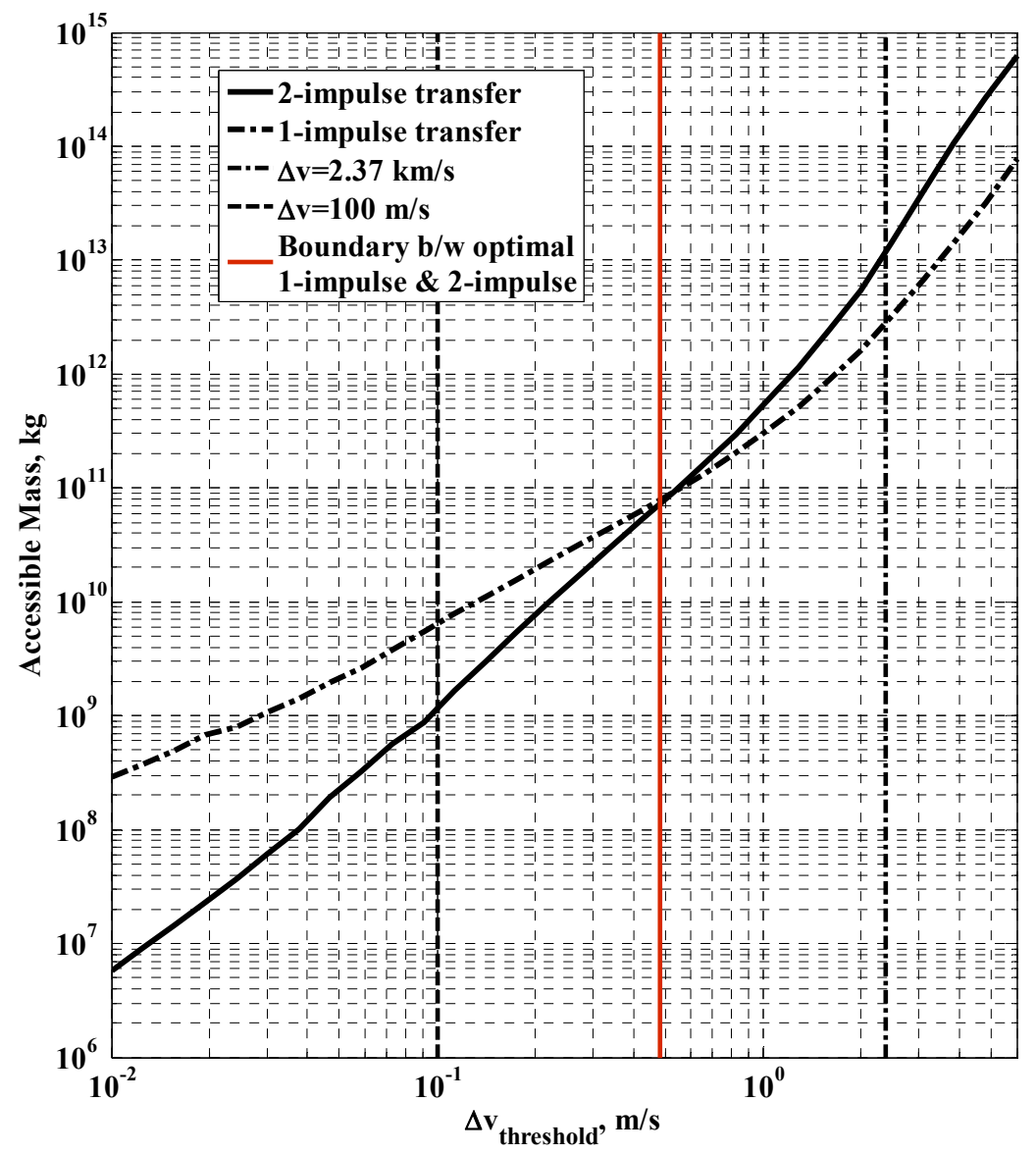

Figure 8: Average accessible asteroid mass for exploitation of resources as a function of $\Delta \mathbf{v}$ threshold.

The results in Figure 8 allow a direct comparison between lunar and asteroid resource exploitation. For a $\Delta v_{t h r}$ equal to the Moon's escape velocity (i.e., $2.37 \mathrm{~km} / \mathrm{s}$ ) the average accessible asteroid material is of the order of $1.75 \times 10^{13} \mathrm{~kg}$ using a two-impulse transfer as described in this paper. Approximately, $3 \times 10^{12} \mathrm{~kg}$ of material could also be captured during Earth fly-by, without having to modify the orbit geometry of these 
objects. Even if the same $\Delta v_{t h r}$ provides access to many orders of magnitude more material at the Moon (i.e., mass of the Moon is $7.3 \times 10^{22} \mathrm{~kg}$ ), the main advantage of asteroid resources with respect lunar resources is that asteroidal material can be exploited at a whole spectrum of $\Delta v$. For example, $6.4 \times 10^{9} \mathrm{~kg}$ of asteroid resources could still be exploited at a $\Delta v_{t h r}$ of only $100 \mathrm{~m} / \mathrm{s}$ by using a serendipitous capture such as the one described by the one-impulse transfer. Lunar material instead requires an energy threshold to overcome the Moon's gravity well (i.e., a $\Delta v$ of $2.37 \mathrm{~km} / \mathrm{s}$ ). On the other hand, the Moon is believed to be a relatively resource-poor body [2], thus asteroid resource exploitation above $2.37 \mathrm{~km} / \mathrm{s}$ may still be an attractive option if the required resource is not easily found at the Moon.

\section{Providing Encounter Opportunities}

Previous sections have assumed that if the orbital intersection exists, then the asteroid would eventually meet the Earth. This statement may be true if the time available to transfer the asteroid is not constrained, but for realistic scenarios this does not occur. Therefore, some analysis on the cost of the maneuvering necessary to ensure the encounter opportunity must be performed.

For a more realistic transfer scenario, in which the orbital phasing is also considered, an additional impulsive maneuvre may be necessary in order to provide the correct phasing to the asteroid. This maneuvre is generally small and must be provided as early as possible, so that the secular effect due to the change in period yields the orbital drift necessary for the asteroid to be at the Earth orbital crossing point at the required time. Hence, if only secular effects are considered [23, 24], which is regarded as a good approximation for the level of accuracy intended in this paper, the phasing maneuvre should correct the difference in mean anomaly $\Delta M$ that exists for the intended encounter (see Figure 3). This is expressed as:

$$
\Delta M=\Delta n \cdot\left(t_{e}-t_{m}\right)
$$

where $\Delta n$ is the change of mean motion of the asteroid due to the phasing maneuvre and $\left(t_{e}-t_{m}\right)$ is the time-span between the maneuvre $\left(t_{m}\right)$ and the encounter (i.e. time at which the Earth is at the crossing point $t_{e}$ ). The change in mean motion of the asteroid can be defined as:

$$
\Delta n=\left(\sqrt{\frac{\mu_{S}}{(a+\delta a)^{3}}}-\sqrt{\frac{\mu_{S}}{a^{3}}}\right)
$$

where $\delta a$ is the change of semi-major axis of the asteroid due to the impulsive manoeuvre. Using the Gauss planetary equations [25], $\delta a$ can be expressed as:

$$
\delta a=\frac{2 a^{2} v_{0}}{\mu_{S}} \delta v_{t}
$$


where $\delta v_{t}$ is the tangential component of the impulsive manoeuvre and $v_{0}$ is the orbital velocity at the point at which the impulsive manoeuvre is applied. Eq.(35) seems to indicate that the optimal position for a phasing manoeuvre is the periapsis, since this is the point at which the orbital velocity $v_{0}$ is maximum. This is generally true, except for cases in which the term $\left(t_{e}-t_{m}\right)$ of equation (33) drives the optimality of the phasing maneuvre.

Finally, rearranging Eq.(33), (34) and (35), the phasing maneuvre necessary to drift the asteroid through $\Delta \mathrm{M}$ angular position at time $t_{e}$, given a impulsive maneuvre at time $t_{m}$, can be expressed as:

$$
\delta v_{t}=\frac{\mu_{S}}{2 a^{2} v_{0}}\left(\left(\frac{\mu_{S}}{\left(\frac{\Delta M}{\left(t_{e}-t_{m}\right)}+\sqrt{\frac{\mu_{S}}{a^{3}}}\right)^{2}}\right)^{1 / 3}-a\right)
$$

which provides a good estimation of the cost of the phasing maneuvre to target an Earth encounter.

Considering an Earth-asteroid configuration such as in Figure 3, an algorithm was implemented that computes the fraction of mean anomalies inside the asteroid orbital path that can be phased with the Earth with a $\delta v_{t}$ smaller than a given threshold. The algorithm requires as an input the $\Delta M$ at a given time $t_{e}$ at which the Earth is assumed to be at the crossing point from which $\Delta M$ is measured. Also a time constraint needs to be specified, which defines the maximum allowed maneuvre time $t_{m}^{\max }$. Then, the algorithm computes the $\delta v_{t}$ necessary to cancel not only the $\Delta M$ gap at time $t_{e}$, but also all other possible encounters opportunities, which are defined by the times at which the Earth is at the crossing points during the time-span available. For each possible encounter two maneuvre times are considered; the first available periapsis passage and $t_{m}^{\max }$. This procedure is repeated for many different angular positions $\Delta M$ at $t_{e}$, from which then the fraction of the orbit that can be phased under a $\Delta v$ limit is calculated.

Figure 9 includes the effect of 40,20 and 10 years time constraints on the accessibility of asteroid resources. The figure also shows accessibility of asteroid material without considering any time constraint (also shown in Figure 8), but this time only the results of the optimal transfer strategy are shown for each $\Delta v$ threshold. From the results in Figure 9 it can be concluded that the free phasing assumption during the description of the transfer models is a good approximation for relatively large $\Delta v$ thresholds. At low $\Delta v$ thresholds some early maneuvering may be required. Note that a 40-year trajectory may not necessarily be envisaged as a trajectory requiring 40 years to be completed. This only suggest the necessity to provide early shepherding maneuvers, allowing asteroids to have the right phasing conditions with Earth. Years later, a short sequence of maneuvers can be provided to achieve a final capture of the asteroid or its mined material. 


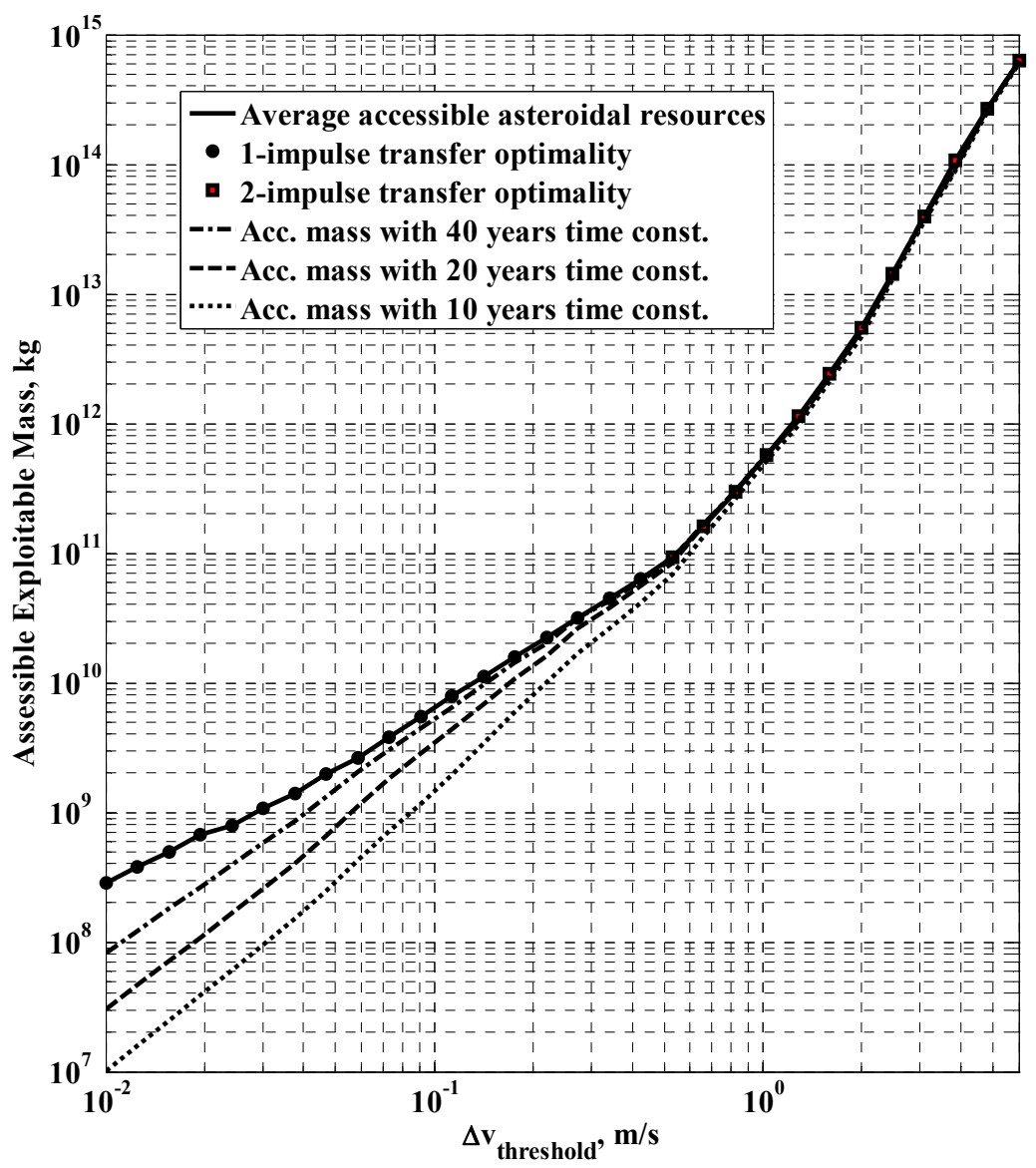

Figure 9: Time constrained and unconstrained average accessible resources.

\section{Discussion}

Figure 8 and 9 do not consider the availability of different materials, nor the number of asteroids that will make up the total resource pool of a given material. The latter issue is of key importance, since if a given resource is spread in a large number of very small objects, gathering all of them may become a cumbersome task, and therefore not economically worthwhile. The question that arises now is how many missions would be required to harvest a given amount of resource. The following sections will discuss about these issues by, first, analyzing the expected size of largest objects to be found within a $\Delta v$ threshold, and second discussing about possible materials and their abundances in the near Earth space.

\section{A. Accessibility of Asteroids}

In order to estimate the average size of each accessible object, we will assume that each single object has the same probability $P$ (see section III.C) to be found in the accessible region. Thus, the probability to find $k$ asteroids within a population of $n$ asteroids in a region delimited by the parameter $\Delta v$ threshold is well described by the binomial distribution (i.e., probability of $k$ successes in $n$ yes/no experiments). In this particular case, for which $P$ is a very low probability and $n$ a very large number of asteroids, Poisson distribution (a limiting case of the binomial distribution when $n$ tends to infinity) represents a very good approximation of the statistical 
behavior of the problem. Therefore, the probability $\mathrm{g}(k, \lambda)$ to find $k$ asteroids when the expected number is $\lambda$ (i.e., $\lambda=P \cdot n)$ can be described by:

$$
g(k, \lambda)=\frac{\lambda^{k} e^{-\lambda}}{k !}
$$

The expected number $\lambda$, or average number of accessible asteroids, can be calculated as:

$$
\lambda\left(D_{\min }\right)=\Delta N\left(D_{\min }<D \leq D_{\max }\right) \cdot P
$$

where $\Delta N$ is the total number of asteroids with diameters larger than $D_{\min }$ and smaller or equal than $D_{\max }$ (Eq.(2)) and $P$ the probability to find objects within a given Keplerian region. In the following, $\Delta N$ will keep $D_{\max }$ fixed to the $32-\mathrm{km}$ diameter, while $D_{\min }$ may vary to modify the value of $\lambda$ as required.

An integration such as:

$$
\int_{n_{N E A}}^{\infty} g(k, \lambda) \cdot d k
$$

yields the probability to find at least $n_{\mathrm{NEA}}$ asteroids when the expected value, or average, is $\lambda$. By finding then the value of $\lambda$ that yields an accumulative probability of $50 \%$, i.e.,

$$
\int_{n_{N E A}}^{\infty} g\left(k, \lambda\left(D_{\min }\right)\right) \cdot d k=0.5
$$

we can estimate the median diameter of the smallest object in the $n_{\mathrm{NEA}}$ set. This procedure can also be repeated with accumulative probabilities of $95 \%$ and $5 \%$ to obtain the $90 \%$ confidence region of the size of the $n_{\mathrm{NEA}}$ smallest object. The results of this procedure can be seen in the following figure. 


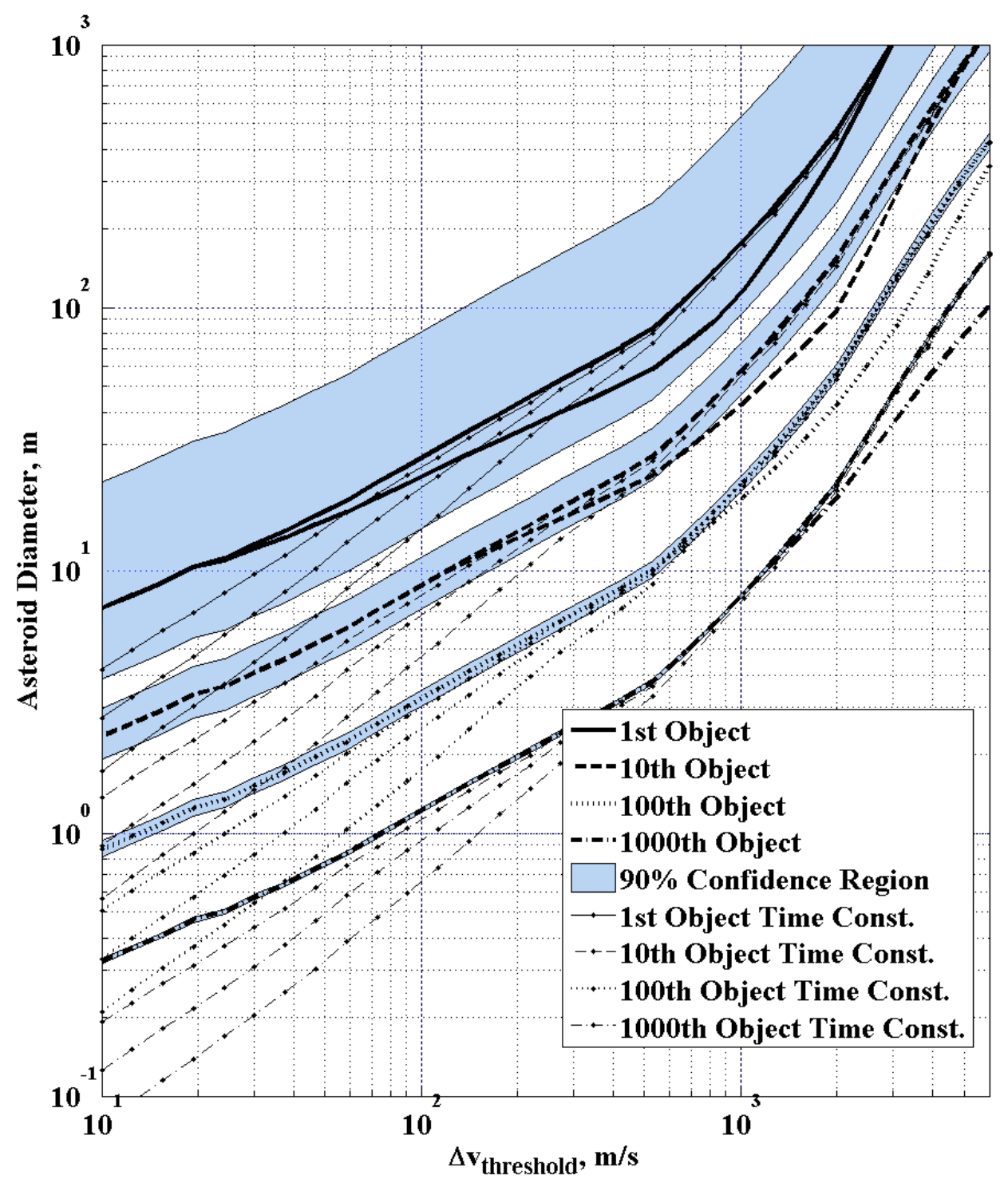

Figure 10: Expected size of the accessible asteroid.

Figure 10 shows the median diameter of the first, tenth, hundredth and thousandth largest accessible asteroid in the near Earth space, together with the $90 \%$ confidence region of each one of these objects. Note that the $90 \%$ confidence regions account only for the statistical uncertainty of finding find $k$ asteroids within a population of $n$ objects, and that the population is assumed to be perfectly described by Eq.(1). The figure also shows the median diameter considering a 2/3 drop in the number of small asteroids as estimated by Harris [18], this is represented by the lower branch in each asteroid set of data. This second population size distribution of asteroids was represented by a four slope power law distribution matching with Eq.(1) at 1-km and 10-m, while providing a $2 / 3$ drop on the accumulative number of asteroids at $100-\mathrm{m}$. Finally, Figure 10 also shows the results by considering 40,20 and 10 years of time constraint in the transfer time, represented as the three departing lines from the main set of data. 
The information in the figure can be read as follows: let us set, for example, the $\Delta v$ threshold at $100 \mathrm{~m} / \mathrm{s}$, the largest accessible object has a 50\% probability to be equal to or larger than 24 meters diameter, while we can say with $90 \%$ confidence that its size should be between 72 meters and 12 meters. We can also see that when accounting for a population of asteroids as estimated by Harris [18] the median becomes 20 meters instead of 24 meters. Finally, we also see that when time constraints are included and phasing maneuvre are estimated as described in section III.D, the median diameter decays to 23 meters when the constraint is set to 40 years, 20 meters if the constraint is 20 years and 13 meters if the limit is at 10 years (solid-dotted lines). The following set of data in the decreasing ordinate axis is the group referring to the 10th largest object found within the region of feasible capture given by a $\Delta v$ threshold of $100 \mathrm{~m} / \mathrm{s}$, whose median diameter is at 8 meters diameter. The 100 th largest object is foreseen to have a diameter of 3 meters and 1000th largest of 1 meter.

The methodology described in this section and the results shown in Figure 10 allow us to attempt now a more speculative discussion about possible resources that may be found and exploited by future space ventures. Asteroids (or comets) in near Earth orbital space include almost all the taxonomical classes found in the main asteroid belt, albeit the relative abundances may experience some differences [26]. Diversity of NEO composition can be inferred by the growing number of categories and sub-categories in the asteroid taxonomical classification [27], as well as by the wide range of meteorite compositions [28]. Thus, the following discussion, which will assume general asteroid abundances and composition that agree with published work [26-29], aims only to provide an order of magnitude estimate for some key resources such as water, metals and semiconductors. The goal of this is to set the ground for a qualitative discussion on the possibilities for future space resource exploitation. Finally, note that in the following sections, in order to keep estimates as conservative as possible, only the taxonomic asteroid class that offers the largest amount of a specific resource is targeted in order to exploit that specific resource.

\section{B. Available Water}

Human beings need approximately 3.5 litres of water per day. Although a large proportion of this water can be recycled by closed-loop life support systems, a proportion of it will be always need to be supplied. Moreover, water can also be used as a propellant for propulsion systems or to provide breathable oxygen for crew once hydrogen and oxygen have been dissociated by hydrolysis. Hence, water is an essential element that any human space mission will need to transport into Earth orbit at the usual launch costs (currently about $\sim 10,000 \$ / \mathrm{kg}$ [30]), unless water could be delivered from near Earth asteroid sources. 
We will now consider hydrated carbonaceous asteroids $(\sim 10 \%$ of the asteroid population [27] with approximate density $\rho_{a}$ of $1300 \mathrm{~kg} / \mathrm{m}^{3}$ [16]) as the only water-carrying objects in the NEA population, and we will also assume that the average fraction of water carried by these objects is about $8.5 \%$ of its weight [29]. With these assumptions, one can easily find an estimated magnitude of the amount of water in near Earth space. Figure 11 shows the water resource pool in the near Earth space. As on Figure 10, the figure can be read as follow; the largest water-carrying asteroid that could be captured at Earth (or that its resources could be transferred to Earth) with, for example, a $\Delta v$ limit of $100 \mathrm{~m} / \mathrm{s}$ could potentially carry 55,000 liters of water. At the same $\Delta v$ limit, the $10^{\text {th }}$ captured object would be carrying carry approximately 2,000 liters of water, the $100^{\text {th }}$ object, instead, would be carrying only 100 liters of water. If, alternatively, the same asteroid diameter is always harvested, thus constant amount of water at each capture, the figure shows that in order to find 10 objects carrying 55,000 liters of water (10-m diameter hydrated carbonaceous asteroid) transfers and capture missions able to provide $500 \mathrm{~m} / \mathrm{s}$ of $\Delta v$ to the asteroids or to its material will have to be planned. It is noted that once an asteroid size or $\Delta v$ region has been depleted it will take millions of years of orbital evolution until the population of asteroids in near Earth space is replenished to the current level [8], thus, in practical terms it would be permanently depleted. These results are quite robust to changes on the assumed density or average fraction of water; in order the results to change in one order of magnitude, the combined change of these two parameters should also change in one order of magnitude. This possibility seems remote, since for example the density could not possibly have a one order of magnitude excursion from the average expected for a $\mathrm{C}$ class asteroid and, on the other hand, the fraction of water assumed here could also be much higher, for example, for extinct comets. There is somehow a higher sensitivity with the abundance of hydrated asteroids ( $\sim 10 \%$ used here), where a factor of 6 change yields 1 order of magnitude change of the results on Figure 10. 


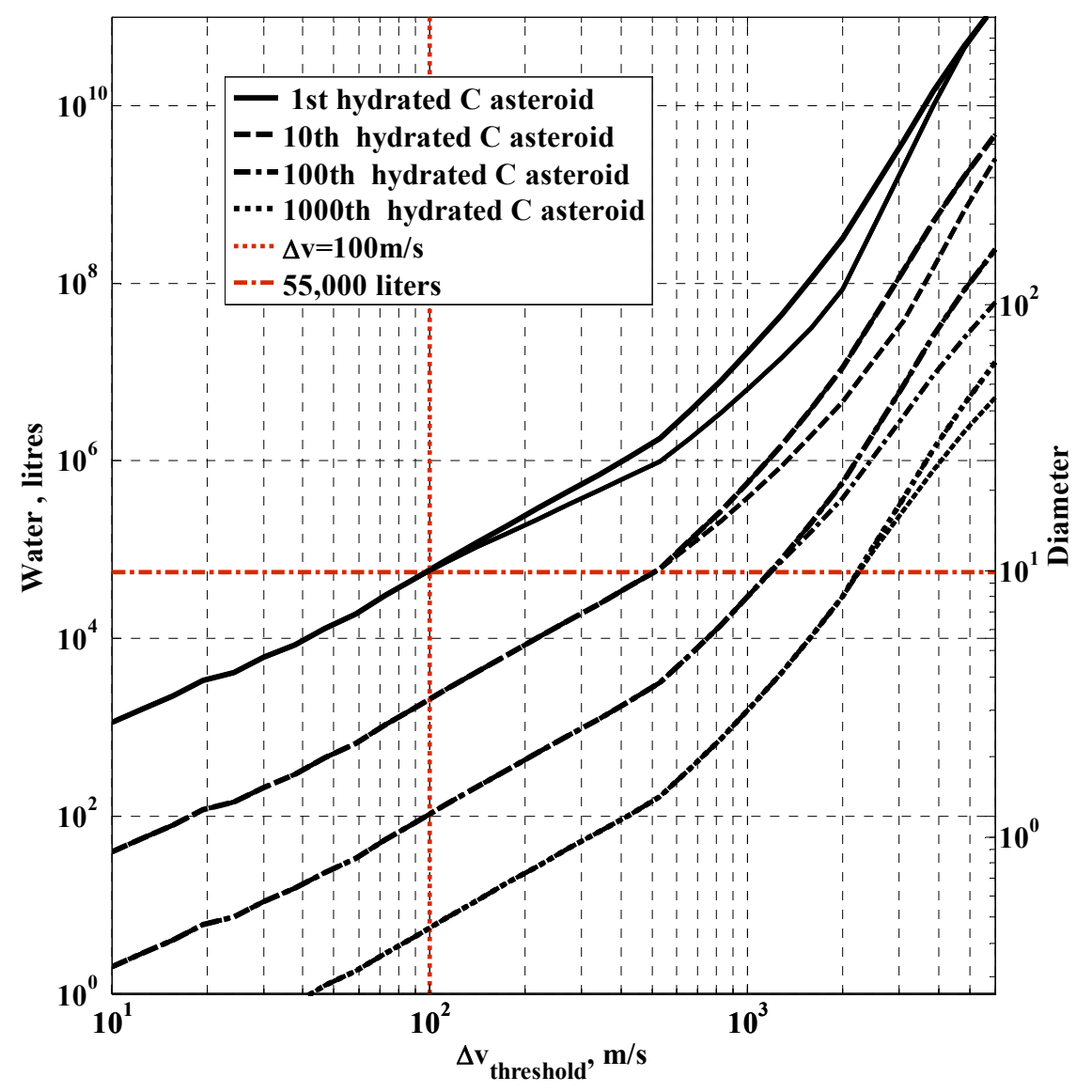

Figure 11: Water Resource Pool. The figure shows the approximate amount of water that could possibly be carried by each water-bearing object.

Note that the $\Delta v$ provided in these resource maps is the change of velocity required for a weakly bound Earth orbit (see section III). If this water should be delivered to Low Earth Orbit (LEO), an approximate transfer of $3.22 \mathrm{~km} / \mathrm{s}$ would still be required. However, from the Earth's surface to LEO, a payload requires typically 9 $\mathrm{km} / \mathrm{s}$ of $\Delta v$, which means that 1 liter of water from the Earth's surface requires an impulse of 9000 Ns to reach LEO. Without considering time constraints to transfer water to the Earth, and hence no phasing maneuvers are accounted for, 2.8 liters of water can reach LEO for the same impulse cost as 1 liter from the Earth's surface. This figure improves radically if the water is transported to the Earth-Moon Lagrangian points and used to fuel interplanetary missions [31]. In terms of invested energy, the NEA population offers a rather large and easily accessible pool of water resources for future human space activities.

\section{Available Metals and Semiconductors}

Similarly to water, metals and semiconductors could also be extracted from asteroids and transferred to Earth orbit for space manufacturing, or extracted once the NEA is captured in Earth orbit. Again, the key issue is the energy cost of manufacturing in space to avoid the large costs associated with launch from Earth. Even for 
processed materials such as silicon wafers for solar cells, which have a high terrestrial manufacturing cost, the cost of manufacturing is still almost an order of magnitude lower than the cost of launching to LEO [32]. Figure 12 shows the approximate amount of iron and silicon resources in near Earth space. Figure 12 assumes that iron is extracted from M-class asteroids (density $\rho_{a}$ of $5300 \mathrm{~kg} / \mathrm{m}^{3}$ [16]), while silicon is extracted from S-class (density $\rho_{a}$ of $2.700 \mathrm{~kg} / \mathrm{m}^{3}$ [16]), where the abundances of these asteroids are taken from Tholen's distribution data in Bus and Binzel [27]. The iron content in an M-class asteroid is assumed to be $88 \%$ of the asteroid mass, while $38 \%$ of the mass of an S-class asteroid is assumed to be silicon dioxide [29], which corresponds to $18 \%$ of silicon in a S-class asteroid. These results show how, for example, within a $\Delta v$ limit of $260 \mathrm{~m} / \mathrm{s}$, an M-class object should be found with a total metal ore of 7,300 tones, the same mass of metal as in the Eiffel tower.

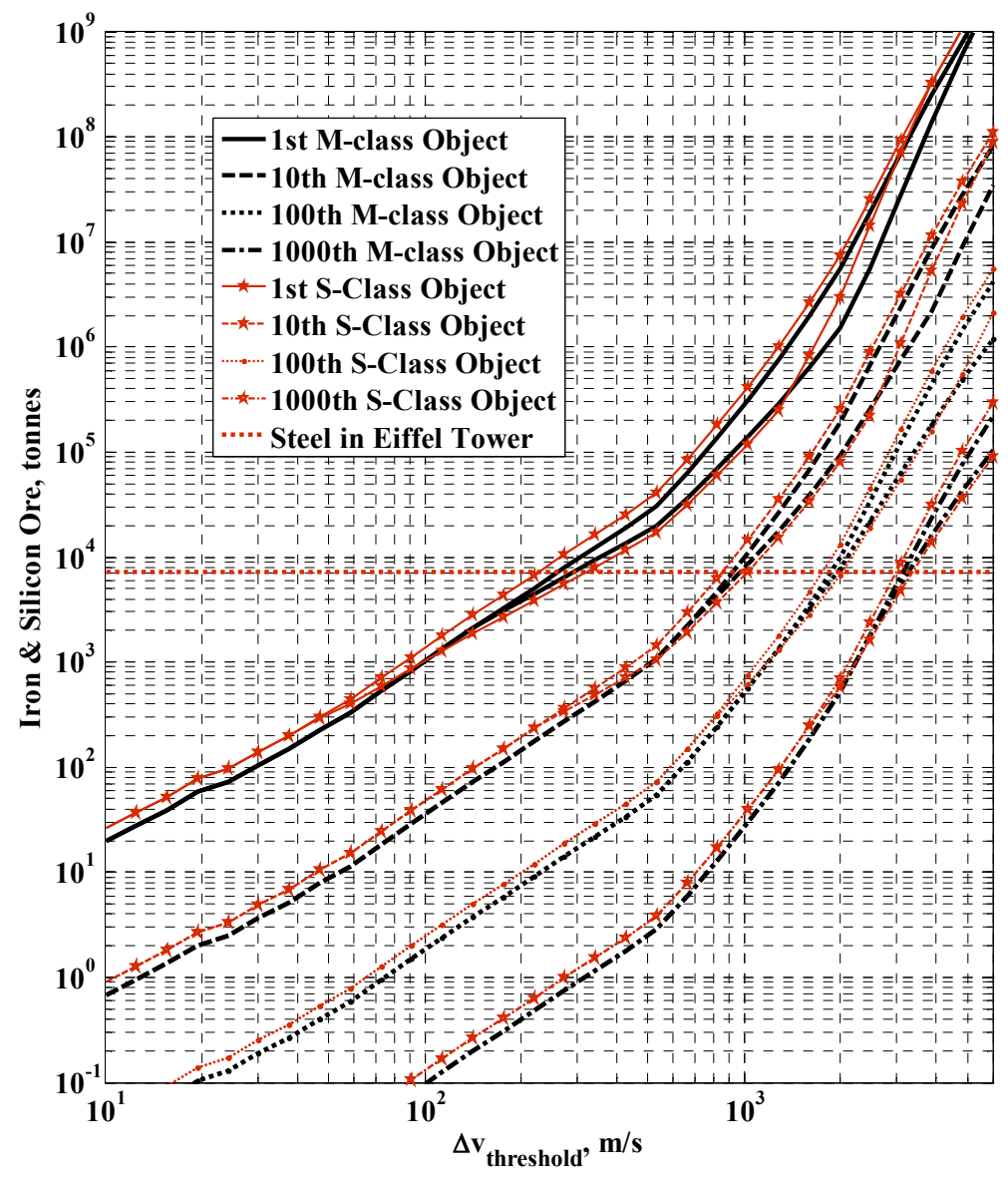

Figure 12: Iron and Silicon resource pool. The figure shows the approximate amount of metal and semiconductor that could be carried by M-class and S-class objects, respectively, found within a $\Delta v$ threshold.

\section{Available Platinum Group Metals}

Unlike metals, such as iron, or semiconductors, such as silicon, which are relatively abundant in the Earth's crust, some rare Earth metals such as the Platinum Group Metals (PGM), which includes ruthenium, rhodium, platinum, palladium, osmium and iridium, could be found in asteroids and may be an important future source of 
these industrial materials. PGMs are of high strategic importance: they have very good catalytic properties, which makes them crucial for the automotive industry and for future hydrogen fuel cells [33]. The record price of platinum is of order $70,000 \$ / \mathrm{kg}$ [34]. At these prices a future scenario in which platinum is extracted from asteroids and transferred to Earth seems plausible. It is still uncertain the amount of PGM that asteroids may have, but there is clear evidence of platinum content on near Earth asteroids, since meteorites have a PGM content [35]. There is some discussion of the fact that S-class asteroids could be a better source of platinum than metallic M-class asteroids. Even though S-class asteroids have a lower concentration of PGM, they are much more abundant than M-class asteroids [36]. Even if that is true, it can be assumed that for PGM mining M-class asteroids are targeted as an obvious metallic source. The platinum content in M-class asteroids is believed to be about $35 \mathrm{ppm}$ [29] and, as previously suggested, about 5\% of asteroids are M-class [27].

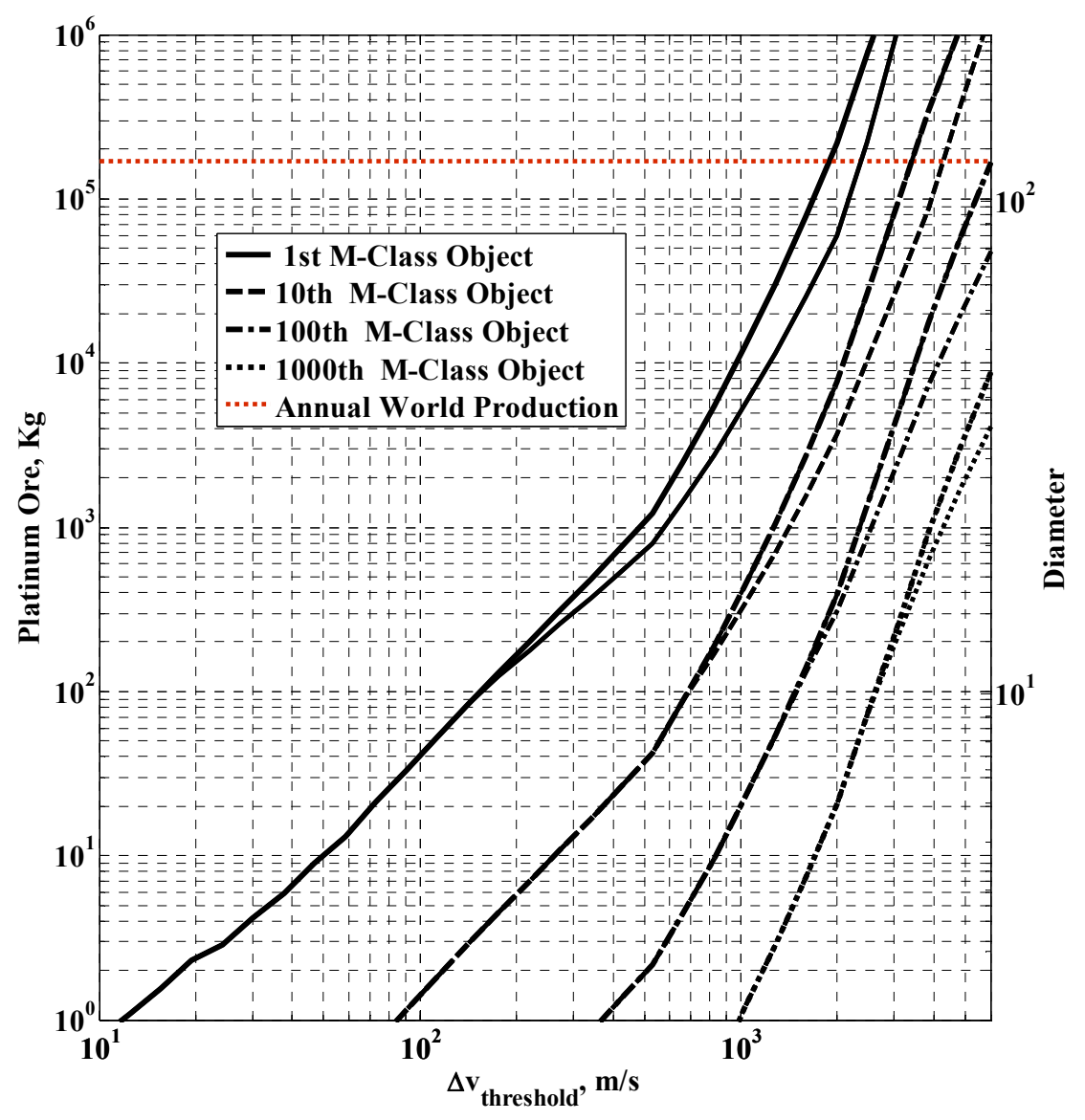

Figure 13: Platinum resource pool. The figure shows the approximate amount of Platinum that could be carried by an $M$ class objects found within a given $\Delta v$ threshold.

Figure 13 shows a plausible scenario for platinum exploitation of asteroids, by assuming the aforementioned concentrations and abundances. It is interesting to note that within a $\Delta v$ threshold of $2 \mathrm{~km} / \mathrm{s}$, it could be possible to capture a metallic asteroid of approximately 110 meters diameter. The platinum content of this asteroid would 
be equivalent to the total Earth production of platinum during one year, which would exceed 6 billion dollars in value $^{5}$. Clearly the economic costs of the transfer and capture would be large, but future demands for PGM may make small-scale demonstration missions attractive.

\section{E. Further Discussion}

As noted previously, asteroids could be mined and their resources transported to the Earth-Moon system. In fact the transport of material could well benefit from the resources found at the asteroid, and, for example, use water as a propellant found in-situ. However, mining operations may entail a technical complexity, both for manned missions and robotic exploration, that may make the possibility of capturing material directly in Earth orbit a desirable option. The possibility of moving the entire asteroid into an Earth bound orbit would allow much higher mission flexibility for resource extraction and transfer operations. In this kind of scenario, concepts for asteroid deflection technologies could be usefully exploited [6]. Although, each asteroid transfer should be carefully design and optimised, by using some of the technologies envisaged for asteroid deflection some general estimations of spacecraft mass in orbit can be provided. For example, Figure 10 shows that a 10-m asteroid could potentially be found with an estimated capture $\Delta v$ of $30 \mathrm{~m} / \mathrm{s}$ using a one-impulse type of transfer. Even such a small object could still supply 50 tonnes of water or 600 tones of metals and PGMs with a possible market value of one million dollars, depending on which type of object it turns out to be (e.g., C, S or M class). An object of this size could be captured during its Earth encounter by providing a collision with a 5-tonne spacecraft at a speed between 4 and $17 \mathrm{~km} / \mathrm{s}$, depending on the actual mass of the asteroid. A kinetic impact scenario like this can be easily envisaged considering that the asteroid would be moving at a speed of $11 \mathrm{~km} / \mathrm{s}$ at the perigee. Even more appealing is the possibility of a ballistic capture of such objects. This 10-m object would be expected to have a relative velocity with the Earth lower than $1 \mathrm{~km} / \mathrm{s}$, which makes it a suitable candidate for a ballistic capture at the Earth by exploiting three-body dynamics [37].

The analysis and results presented in this paper are intended to provide a qualitative analysis on the feasibility of asteroid exploitation. The results and subsequent discussion have only drawn the 'big picture' for future asteroid resource utilization. The hypothesis from which this work is based have tried to be as conservatives as possible, so that the real accessible mass should be expected to be higher. For example, the transfer models provided a conservative, worst case scenario for the required $\Delta \mathrm{v}$, and so the available asteroid mass found is then a lower limit. More complex trajectories, such as multiple Earth fly-by, lunar gravity assists or manifold dynamics, would be expected to provide a significant increase in captured mass. However, some

\footnotetext{
${ }^{5} \mathrm{http}: / /$ www.platinum.matthey.com/
} 
hypothesis will require of future work to completely assess their significance. For example, the assumption on the orbital distribution being independent of the asteroid size [8]. Non-gravitational perturbations affect objects of different size differently, which implies that the different asteroid sources may be supplying different asteroid size distributions, since non-gravitational perturbations are the main mechanisms that feed the different asteroid sources. For the same reason, different orbital regions may contain a higher population of a given type of asteroids (i.e., different composition). Despite these possible sources of inaccuracy, the results shown in the paper should still hold their qualitative value.

\section{Conclusion}

This paper has shown that the utilization of asteroid resources may be a viable means of providing substantial mass in Earth orbit for future space ventures. A statistical population of near Earth asteroids has been used, along with a map of the Keplerian orbital element space from which the Earth can be reached under a given series of impulsive manoeuvres, to estimate the statistical amount of accessible asteroid resources within a given specific transfer energy. It has been shown by this method that interesting asteroids with diameter of order tens of meters to a few hundred meters diameter can likely be found in orbits such that the energetic requirements to transport their resources back to Earth will be very low. The utilization of these potential resources could play an important role in future space undertakings, reducing the energy requirements for space transportation. An interesting characteristic of asteroid utilization is that allows access to resources at an incremental level of energy, while lunar resources require always a minimum threshold equal to the Moon's escape velocity. It has been shown in the paper, that there is a reasonable amount of accessible resources with transfer energies lower than those required to exploit the Moon. Exploitation of higher energy transfers may only be justifiable if the required resource is not available on the Moon.

Finally, the size distribution of objects for near-Earth objects ensures that the amount of exploitable mass is primarily made up of the largest objects within Earth reach. This guarantees that most of the exploitable mass could be successfully harvested by only a few mining or capture missions. Small objects with a diameter of order tens of meters to a few hundred meters diameter could potentially be the first targets for strategic resources.

\section{Acknowledgments}

We thank William Bottke for kindly providing us with the NEA distribution data. The work reported was supported by European Research Council grant 227571 (VISIONSPACE). 


\section{References}

[1]. Mckay, M. F., Mckay, D. S., and Duke, M. B. "Space Resources: Scenarios," NASA-SP-509. Vol. 1, NASA, Lyndon B. Johnson Space Center. Houston, Texas, 1992.

[2]. Lewis, J. S. Mining the sky: untold riches from asteroids, comets and planets: Helix Books/Perseus Books 1996.

[3]. Chapman, C. R. "The hazard of near-Earth asteroid impacts on Earth," EPSL Frontiers Vol. 2, 2004, pp. 1-15. Doi: 10.1016/j.eps1.2004.03.004

[4]. Kawaguchi, J., Fujiwara, A., and Uesugi, T. "Hayabusa-Its technology and science accomplishment summary and Hayabusa-2," Acta Astronautica Vol. 62, No. 10-11, 2008, pp. 639-647. Doi: 10.1016/j.actaastro.2008.01.028

[5]. Barucci, M., Yoshikawa, M., Michel, P., Kawagushi, J., Yano, H., Brucato, J., Franchi, I., Dotto, E., Fulchignoni, M., Ulamec, S., and Marco Polo Science, T. "MARCO POLO: near earth object sample return mission," Experimental Astronomy Vol. 23, No. 3, 2009, pp. 785-808. Doi: 10.1007/s10686008-9087-8

[6]. Sanchez, J. P., Colombo, C., Vasile, M., and Radice, G. "Multi-criteria Comparison among Several Mitigation Strategies for Dangerous Near Earth Objects," Journal of Guidance, Control and Dynamics Vol. 32, No. 1, 2009, pp. 121-142. Doi: 10.2514/1.36774

[7]. Stokes, G. H., Yeomans, D. K., Bottke, W. F., Chesley, S. R., Evans, J. B., Gold, R. E., Harris, A. W., Jewitt, D., Kelso, T. S., McMillan, R. S., Spahr, T. B., and Worden, P. "Study to Determine the Feasibility of Extending the Search for Near-Earth Objects to Smaller Limiting Diameters." NASA, 2003.

[8]. Bottke, W. F., Morbidelli, A., Jedicke, R., Petit, J.-M., Levison, H. F., Michel, P., and Metcalfe, T. S. "Debiased Orbital and Absolute Magnitude Distribution of the Near-Earth Objects," Icarus Vol. 156, No. 2, 2002, pp. 399-433. Doi: 10.1006/icar.2001.6788

[9]. L.W.Alvarez, W.Alvarez, F.Asaro, and H.V.Michel. "Extraterrestrial Cause for the Cretaceous-Tertiary Extinction," Science Vol. 208, No. 4448, 1980, pp. 1095-1108. Doi: 10.1126/science.208.4448.1095

[10]. Harris, A. W. "What Spaceguard did," nature Vol. 453, 2008, pp. 1178-1179. Doi: 10.1038/4531178a

[11]. Ivezic, Z., Tyson, J. A., Juric, M., Kubica, J., Connolly, A., Pierfederici, F., Harris, A. W., Bowell, E., and Collaboration, L. "LSST: Comprehensive NEO Detection, Characterization, and Orbits," Near 
Earth Objects, Our Celestial Neighbors: Opportunity And Risk Vol. 236, 2007, pp. 353-362. $10.1017 / \mathrm{S} 1743921307003420$

[12]. Veres, P., Jedicke, R., Wainscoat, R., Granvik, M., Chesley, S., Abe, S., Denneau, L., and Grav, T. "Detection of Earth-impacting asteroids with the next generation all-sky surveys," Icarus Vol. 203, No. 2, 2009, pp. 472-485. Doi: 10.1016/j.icarus.2009.05.010

[13]. Morbidelli, A., Bottke, W. F., Froeschlé, C., and Michel, P. "Origin and Evolution of Near-Earth Objects," Asteroids III. University of Arizona Press, Tucson, 2002, pp. 409-422.

[14]. Struck, C. "The Feasibility of Shading the Greenhouse with Dust Clouds at the Stable Lunar Lagrange Points," J. British Interplanetary Soc Vol. 60, 2007, pp. 82-89.

[15]. Bewick, R., Sanchez, J. P., and McInnes, C. "An L1 positioned dust cloud as an effective method of space-based geo-engineering," 61st International Astronautical Congress Vol. IAC-10.D1.1.7,Prague, Czech Republic, 2010, p. 14.

[16]. Chesley, S. R., Chodas, P. W., Milani, A., and Yeomans, D. K. "Quantifying the Risk Posed by Potential Earth Impacts," Icarus Vol. 159, 2002, pp. 423-432. Doi: 10.1006/icar.2002.6910

[17]. Steel, D. "Tunguska at 100," Nature Vol. 453, 2008, pp. 1157-1159. Doi: 10.1038/4531157a

[18]. Harris, A. W. "An Update of the Population of NEAs and Impact Risk," Bulletin of the American Astronomical Society Vol. 39, 2007, p. 511.

[19]. Stuart, J. S. "Observational constraints on the number, albedos, size, and impact hazards of the nearEarth asteroids." Massachusetts Institute of Technology, 2003, p. Thesis (Ph. D.).

[20]. Opik, E. J. "Collision Probabilities with the Planets and the Distribution of Interplanetary Matter," Proceedings of the Royal Irish Academy. Section A: Mathematical and Physical Sciences Vol. 54, 1951/1952, pp. 165-199.

[21]. Jack, G. H., and Goda, M. P. "The Fragmentation of Small Asteroids in the Atmosphere," The Astronomical Journal Vol. 105, No. 3, 1993, pp. 1114-1144. Doi: 10.1086/116499

[22]. Bonanno, C. "An analytical approximation for the MOID and its consequences," Astronomy and Astrophysics Vol. 360, 2000, pp. 411-416.

[23]. Scheeres, D. J., and Schweickart, R. L. "The Mechanics of Moving Asteroids," Planetary Defense Conference, Orange County, California, 2004. Paper AIAA-2004-1446 
[24]. Izzo, D. "Optimization of Interplanetary Trajectories for Impulsive and Continuous Asteroid Deflection," Journal of Guidance, Control and Dynamics Vol. 30, No. 2, 2007, pp. 401-408. Doi: $10.2514 / 1.21685$

[25]. Battin, R. H. Introduction to the Mathematics and Methods of Astrodynamics Reston, Virginia: American Institute of Aeronautics and Astronautics, 1999.

[26]. Binzel, R. P., Lupishko, D. F., Martino, M. D., Whiteley, R. J., and Hahn, G. J. "Physical Properties of Near-Earth Objects," Asteroids III. University of Arizona, Tucson, Arizona, 2002, pp. 255-271.

[27]. Bus, S. J., and Binzel, R. P. "Phase II of the Small Main-Belt Asteroid Spectroscopic Survey," Icarus Vol. 158, 2002, pp. 146-177. Doi: 10.1006/icar.2002.6856

[28]. Lewis, J. S., and Hutson, M. L. "Asteroidal Resource Opportunities Suggested by Meteorite Data," Resources of Near-Earth Space. University of Arizona Press, Tucson Arizona, 1993, pp. 523 - 542.

[29]. Ross, S. D. "Near-Earth Asteroid Mining." CA: Department of Control and Dynamical Systems, Pasadena, 2001, pp. 1-24.

[30]. James, R. W., and Wiley, J. L. Space Mission Analysis and Design: Microcosm Press, 2003.

[31]. Martin, W. L., and Shane, D. R. "The Lunar L1 Gateway: Portal to the Stars and Beyond," AIAA Space 2001 Conference. Albuquerque, New Mexico, 2001.

[32]. Mauk, M. G. "Silicon solar cells: Physical metallurgy principles," Journal of the Minerals, Metals and Materials Society Vol. 55, No. 5, 2003, pp. 38-42. Doi: 10.1007/s11837-003-0244-2

[33]. Committee on Critical Mineral Impacts on the U.S. Economy. "Minerals, Critical Minerals and the U.S. Economy ". THE NATIONAL ACADEMIES PRESS, Washington, 2001.

[34]. Crandall, W. B. "Abundant Planet: Enabling Profitable Asteroid Mining." Abundant Planet 501(c)3 organization, Redwood City, CA 94062, 2009, pp. 1-23.

[35]. Nichiporuk, W., and Brown, H. "Platinum and Iridium abundances in Meteorites," Science Vol. 9, No. 6, 1962, pp. 245-246.

[36]. Crandall, W. B. "Asteroid population, characterization, and accessibility," The Wealth of Asteroids: Incorporating Near-Earth Resources into the Human Economy. Abundant Planet, Redwood City, CA 94062, 2009.

[37]. Kemble, S. "Interplanetary Missions Utilising Capture and Escape Through the Lagrange Points," 54th International Astronautical Congress Vol. IAC-03-A.1.01,Bremen, Germany, 2003. 
